\title{
TEXTO Y CONTEXTO. EL MUSEO NACIONAL DE ARTES DECORATIVAS COMO FUENTE EN LA INVESTIGACIÓN HISTÓRICO-EDUCATIVA ${ }^{\alpha}$
}

\author{
Text and context. The National Museum of Decorative Arts \\ as a source for the research in the History of Education
}

\section{María Villalba Salvador ${ }^{\circledR}$}

Fecha de recepción: 01/10/2018 • Fecha de aceptación: 16/12/2018

Resumen. Este artículo estudia las correspondencias y paralelismos que existen en el contexto de creación del Museo Nacional de Artes Decorativas, antes Museo Nacional de Artes Industriales (MNAD), en la segunda mitad del siglo XIX, con el Museo Pedagógico Nacional y los principios de la Institución Libre de Enseñanza. Señala las conexiones y semejanzas en cuanto a principios y actuaciones en los comienzos y en la misión de las dos instituciones, así como los referentes en el contexto europeo y español. Desde la constancia de numerosas coincidencias en planteamientos, modos de proceder y elementos coincidentes en las colecciones, se propone finalmente una vía de trabajo que ponga en valor la colección del MNAD y las piezas custodiadas en instituciones relacionadas con las artes aplicadas — bien sean museos o casas museo-, y las de los museos pedagógicos, para el aprendizaje y la enseñanza de la Historia el Arte y de la Historia de la Educación en España. Contemplar con la perspectiva del paso del tiempo el paralelismo entre los dos museos, produce una foto significativa de una parte de los intereses educativos y sociales del primer tercio del siglo XX en España.

Palabras clave: Museo Nacional de Artes Decorativas; Museo Pedagógico Nacional; Artes decorativas; Museos industriales; Museos pedagógicos; Educación en museos; Historia del arte.

\footnotetext{
a Agradezco a Isabel Rodríguez Marco y a Ana Cabrera Lafuente, Conservadoras del Museo Nacional de Artes Decorativas y del Museo del Traje, por su gran ayuda, y a Sofía Rodríguez Bernis, directora del MNAD, por las informaciones facilitadas. También a la profesora Carmen Blanco Jiménez, de la Universidad Autónoma de Madrid, por la orientación y estímulo al inicio de este trabajo.

${ }^{\text {B }}$ Departamento de Didácticas Específicas. Facultad de Formación de Profesorado y Educación. Universidad Autónoma de Madrid. c/ Francisco Tomás y Valiente, 3. Ciudad Universitaria de Cantoblanco, 28049, Madrid. España. maria.villalba@uam.es.
}

Cómo citar este artículo: Villalba Salvador, María. «Texto y contexto. El Museo Nacional de Artes Decorativas como fuente en la investigación histórico-educativa». Historia y Memoria de la Educación 10 (2019): 271-308 
Abstract. This article considers the parallels and the relationships between the context of creation of the current National Museum of Decorative Arts (Spain), formerly National Museum of Industrial Arts, in the second half of the XIX century, together with the National Pedagogical Museum and the principles of the Institución Libre de Enseñanza (Free Teaching Institution). It highlights the connections and parallels with regard to the principles and actions of the two institutions at their beginnings and in their missions, as well as drawing comparisons with other cases in the Spanish and European contexts. Based on the evidence that there are numerous coincidences in many of the collections' approaches, procedures and elements, we propose a line of work that takes into account the collections of institutions related to applied arts \either museums or home

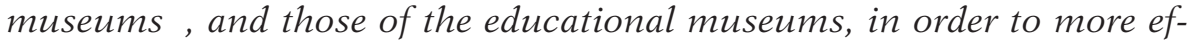
fectively teach Art History and the History of Education in Spain. Looking back into the past to the parallels between the two museums can provide a meaningful picture of part of the educational and social interests of the first third of the XX century.

Keywords: National Museum of Decorative Arts (Spain); National Pedagogical Museum (Spain); Decorative arts; Industrial museums; Pedagogical museums; Art history; Museum education.

\section{INTRODUCCIÓN. ENTORNO DE SITUACIÓN Y FECHAS CLAVE}

La década de los años setenta del siglo XIX presenta dos acontecimientos cruciales para este trabajo: la creación de un Museo Industrial en 1871 y la fundación de la Institución Libre de Enseñanza en 1876, un proyecto pedagógico cuya pretensión era transformar de la sociedad española mediante la educación. Pocos años faltaban para la creación del Museo de Instrucción Primaria (después Museo Pedagógico Nacional) en 1882, que sería modelo para otras instituciones de la misma tipología.

Este artículo aborda el estudio del entorno de creación del Museo Nacional de Artes Decorativas (en adelante MNAD) y las conexiones que se pueden establecer en su origen entre esta institución, los museos pedagógicos y, en concreto, el Museo Pedagógico Nacional (MPN), y los planteamientos de la Institución Libre de Enseñanza (ILE). De ellas se suscitan algunas propuestas de carácter educativo para la puesta en 
valor de las colecciones de dicho museo y la dinamización de las colecciones de los museos pedagógicos.

Hoy en día los principios de la ILE permanecen y se hacen presentes de muy diversas formas. Así, la exposición denominada El arte de saber ver, organizada por Acción Cultural Española (AC/E) y la Fundación Francisco Giner de los Ríos [Institución Libre de Enseñanza], ${ }^{1}$ con sede en Madrid y en Toledo (2016-2017), mostraba la importancia concedida por sus miembros a la enseñanza de la historia del arte desde un planteamiento de experimentación y, en ese sentido, la necesidad de tener en cuenta los museos como referentes y objetos de trabajo. Sobre ellos, y sobre la contemplación directa de la obra de arte y el entorno, Manuel B. Cossío (1857-1935) proyectaba el «arte de saber ver». El panel explicativo que daba entrada a la exposición reproducía parte del texto escrito por Cossío en 1879:

El mundo entero debe ser, desde el primer instante, objeto de atención y materia de aprendizaje para el niño, como lo sigue siendo, más tarde, para el hombre. Enseñarle a pensar en todo lo que le rodea y hacer activas sus facultades racionales es mostrarle el camino por donde se va al verdadero conocimiento, que sirve después para la vida. Educar antes que instruir [...], es el ideal que aspira a cumplir, mediante ese arte de saber ver, la pedagogía moderna. $^{2}$

Se trataba de empezar por los cimientos, por la educación, de formar individuos, no sólo de instruirlos, siguiendo las ideas del krausismo. Para Cossío era esencial contribuir a crear una sensibilidad artística en sus alumnos, hablaba de la enseñanza del arte como desarrollo del gusto, de la emoción estética y del sentimiento de lo bello. El arte, en todas sus manifestaciones, está relacionado con el pueblo que lo crea y hay que saberlo ver, intuir y captar directamente desde los primeros años escolares. De su pensamiento destaca una idea básica: el hombre debe

\footnotetext{
${ }^{1}$ Celebrada en la sede de la Institución Libre de Enseñanza, en Madrid, del 30 de noviembre de 2016 al 23 de abril de 2017.

2 Manuel B. Cossío, «Carácter de la pedagogía contemporánea. El arte de saber ver», BILE, año III, núms. 65 y 66, 31 de octubre y 16 de noviembre de 1879. Recogido en Manuel B. Cossío, De su jornada (fragmentos) (Madrid: Imprenta de Blass, 1929), 3-12: así aparece reseñado en El arte de saber ver. Manuel B. Cossío. La Institución Libre de Enseñanza y El Greco, ed. Salvador Guerrero (Madrid: Fundación Francisco Giner de los Ríos, Institución Libre de Enseñanza, 2016), 357-358.
} 
recibir de ese gran depósito artístico cultural que se ha ido acumulando durante siglos, una completa educación estética.

Además, los institucionistas fueron absolutamente pioneros en España en la defensa tanto de las artes decorativas e industriales como de las artes populares, y de su enorme potencial educativo. Entre los numerosos viajes que realizó Cossío para asistir a congresos, conferencias, visitas a museos e instituciones educativas, merece destacar que durante su viaje a Londres en agosto de 1884 a la Conferencia Internacional de Educación de South Kensington, conoció las teorías de Ruskin, ${ }^{3}$ y de su intervención valoró fundamentalmente lo relativo a la educación del sentimiento para alcanzar la comprensión del arte. ${ }^{4}$ También percibió el trabajo de las escuelas de South Kensington en relación con la formación técnica y la educación del gusto artístico. ${ }^{5}$ Todo ello le acercaría al movimiento Arts and Crafts, lo que le hizo darse cuenta de la importancia del trabajo manual que reforzaba cualidades artísticas y morales. ${ }^{6}$

También lo reflejaba la exposición El arte de saber ver en una sección especial, ${ }^{7}$ donde se exponían trabajos manuales en tejidos, cerámica y loza esmaltada, entre otros, transmitiendo el afán por recuperar la tradición y las creaciones populares, tan importantes para la conservación del patrimonio cultural español.

\footnotetext{
3 Sobre este autor, véase Lionello Venturi, Historia de la crítica de arte (Barcelona: Gustavo Gili, 1979), 183-190; Mireia Freixa (ed.), Las vanguardias del siglo XIX (Barcelona: Gustavo Gili, 1982), 151-154; Ian Chilvers y Harold Osborne, The Oxford Dictionary of Art (New York: Oxford University Press, 1997), 495-496; Joshua C. Taylor, Nineteenth-Century Theories of Art (Berkeley and Los Angeles, California: University of California Press, 1987), 286-296; John Ruskin, Las piedras de Venecia y otros ensayos sobre arte (Barcelona: Biblok Book Export, 2016).

${ }^{4}$ Eugenio Otero Urtaza, Manuel Bartolomé Cossío. Trayectoria de un educador (Madrid: Publicaciones de la Residencia de Estudiantes y Consejo Superior de investigaciones Científicas, 1994), 143 148, y Manuel B. Cossío y Santos M. Robledo, Anuario de primera enseñanza correspondiente a 1886 (Madrid: Imprenta del Colegio Nacional de Sordomudos, 1887), 315.

${ }^{5}$ Otero, Manuel Bartolomé Cossío, 148; Cossío y Robledo, Anuario de primera enseñanza correspondiente a 1886, 315 .

' Eugenio Otero Urtaza, «Los viajes de formación de Cossío entre 1879 y 1889», en El arte de saber ver. Manuel B. Cossío. La Institución Libre de Enseñanza y El Greco, ed. Salvador Guerrero (Madrid: Fundación Francisco Giner de los Ríos, Institución Libre de Enseñanza, 2016), 179.

7 Sofía Rodríguez Bernis, "Cossío y las artes populares», en Manuel B. Cossío. La Institución Libre de Enseñanza y El Greco, ed. Salvador Guerrero (Madrid: Fundación Francisco Giner de los Ríos, Institución Libre de Enseñanza, 2016), 181-191.
} 


\section{PROGRESIVO INTERÉS HACIA LAS ARTES INDUSTRIALES EN EL} SIGLO XIX

Con carácter previo a estos años de la década de los setenta de los que hablaba más arriba, durante el siglo XIX se sucedieron cambios sociales y políticos de envergadura, que transformaron nuestro país y supusieron un desarrollo decisivo de la burguesía. En el contexto de la década moderada en España (1844-1854) tuvieron lugar intentos de desarrollo industrial y de creación de un estado centralista, liberal y burgués. Madrid se perfilaba como centro administrativo y jerárquico del país y hubo una primera iniciativa de creación de un museo de artes industriales, entendido como una especie de depósito de muestrarios de nuestros productos fabriles y un estímulo de competencia para el perfeccionamiento de la industria y de las artes. Todo esto sucedía en 1851, para proteger la industria y el comercio nacional, y difundir en el extranjero nuestros progresos, pero la empresa no llegó a término. ${ }^{8}$ Esta primera intentona se situaba, asimismo, teniendo como fondo la celebración de la Exposición Internacional de Artes Decorativas e Industriales Modernas del mismo año, cuando en el panorama internacional se pretendía dar un sentido estético y educativo a la industrialización, y se planteaba la formación de fabricantes, diseñadores y consumidores. Una época en la que se sucedieron estilos y planteamientos artísticos que afectaron de lleno a las artes decorativas.

Por otra parte, en la misma década se promulgó la Ley Moyano o Ley de Instrucción Pública de 1857. Con ella se dio nuevo impulso a las enseñanzas profesionales mediante las Escuelas de Artes y Oficios. ${ }^{9}$ Y es precisamente a la primera de ellas, la de Madrid, a la que se vincula la segunda tentativa de creación de un museo industrial en 1871 durante el reinado de Amadeo de Saboya. Fue a instancias de Ruiz Zorrilla, Ministro de Fomento, cuando se creó dicho museo, pero sin dotación explícita. ${ }^{10}$ Los pasos fueron los siguientes: se firmó el decreto para la creación de la Escuela de Artes y Oficios, que defendía el interés y trascendencia

\footnotetext{
${ }^{8}$ Ana Cabrera Lafuente y María Villalba Salvador, «El Museo Nacional de Artes Decorativas (Madrid). De Museo Industrial a Museo Nacional de Artes Industriales (1850-1912). Antecedentes para la historia del Museo», RdeM. Revista de Museología 30-31 (2004): 81-88.

9 Ana María Montero Pedrera, «Origen y desarrollo de las Escuelas de Artes y Oficios», Historia de la Educación 17 (1998): 319-321.

${ }_{10}$ Cabrera y Villalba, «El Museo Nacional de Artes Decorativas», 81-88.
} 
de la educación e instrucción del artesano y el obrero, en beneficio del progreso del país. La escuela se adscribía al Conservatorio de Artes, ${ }^{11}$ institución fundada en 1824 siguiendo el modelo francés para mejorar «las operaciones industriales, tanto en las artes y los oficios, como en la agricultura».12 En el mismo documento y vinculado a la Escuela de Artes y Oficios se creaba también un Museo Industrial. ${ }^{13}$ Sin embargo, ambas ideas, aprobadas legalmente en 1851 y 1871, quedaron en proyectos de carácter teórico, aunque no se les puede restar importancia pues destacan el interés de España en crear una institución de ese perfil, siguiendo el impulso de las Exposiciones Universales y la creación de otros museos similares en Europa.

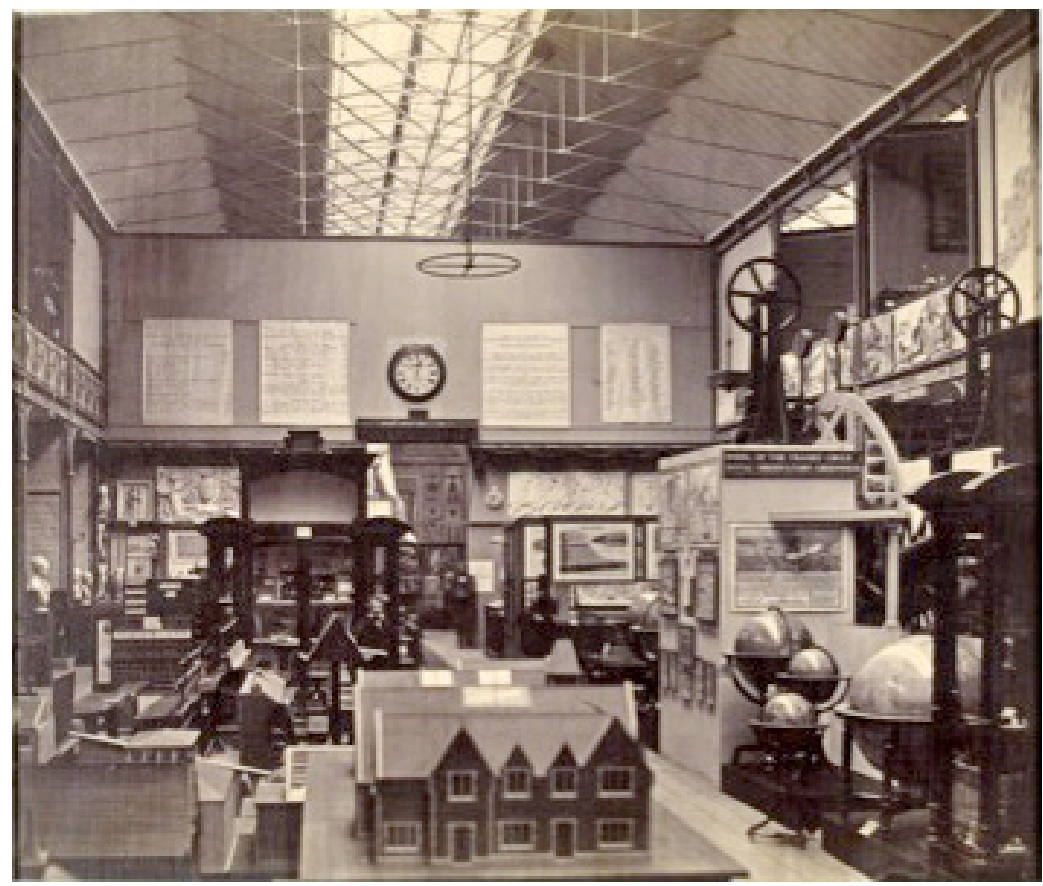

Figura 1. Interior de la Sección de Educación del South Kensington Museum, ('the Brompton Boilers'). Inv. 32055 ca. 1859 @ Victoria and Albert Museum, London.

11 «Real Decreto de 5 de mayo de 1871», Gaceta de Madrid (8 de mayo de 1871):1-3.

12 Jean-Louis Guereña y Alejandro Tiana, «La educación popular», en Historia de la Educación en España contemporánea. Diez años de investigación, eds. Jean-Louis Guereña, Julio Ruiz Berrio, Alejandro Tiana Ferrer (Madrid: M.E.C., 1993), 146.

13 “Art. $7^{\circ}$ del «Real Decreto de 5 de mayo de 1871», Gaceta de Madrid (8 de mayo de 1871): 2. 
Realmente en aquellos años de la mitad del siglo XIX surgían, como señala García del Dujo, ${ }^{14}$ los primeros museos de educación. En 1851 y tras la Exposición Internacional de Artes Decorativas e Industriales Modernas, citada más arriba, en Alemania se creaba una sección educativa en el Museo de la Industria de Stuttgart, para mejorar la enseñanza con aplicaciones industriales. Casi al tiempo, y como consecuencia de la gran muestra londinense, Inglaterra ponía en marcha la creación del South Kensington Museum (SKM), del que pasó a formar parte el Museo de Manufacturas (1837), fundado para instruir al público en todas las materias relativas al diseño, crear demanda e incrementar la idiosincrasia de las manufacturas nacionales. ${ }^{15}$ El planteamiento educativo de esta nueva institución era tan evidente que no tardó mucho en crearse una sección denominada The Educational Museum (1857) que presentaba, entre otras secciones, una «Biblioteca de Arte Circulante»; modelos ilustrativos de métodos de construcción; modelos utilizados en educación y maquetas arquitectónicas (figura 1), que incluían obras de arte y que, en el caso de los tejidos, se trataba de modelos repetidos o muy similares a los de la colección. Henry Cole declaró que debía ser una escuela para todo el mundo y muchos de los objetos coincidían con los propios de los museos pedagógicos y los de reproducciones artísticas (yesos, dibujos, láminas, modelos). ${ }^{16}$ El programa educativo formaba parte de los precoces principios fundacionales: trabajaba desde los inicios la didáctica y la guía de 1865 sugería una especie de bazar pedagógico. En definitiva, se trataba de un museo muy comprometido con la educación (figura 2 ). Su creación y puesta en marcha ${ }^{17}$ le convirtieron en referencia en materia museística y educativa en Europa, como demuestran recientes investigaciones, que desvelan debates sobre la

\footnotetext{
14 Ángel García del Dujo, «El museo pedagógico nacional y las corrientes pedagógicas contemporáneas», Historia de la Educación. Revista Interuniversitaria 4 (1985): 173-176. http://revistas.usal.es/ index.php/0212-0267/article/view/6622, (última consulta 10-08-2018).

15 Victoria \& Albert Museum, https://www.vam.ac.uk/articles/building-the-museum (última consulta 20-08-2018).

${ }^{16}$ Giles Waterfield, «The People`s Galleries. South Kensington Museum - V\&A», https://yalebooksblog. co.uk/2015/09/03/the-peoples-galleries-south-kensington-museum-the-va/ (última consulta 20-08-2018).

${ }_{17}$ Clive Wainwright, (edited for publication by Charlotte Gere), «The making of South Kensington Museum I. The Governnment Schools of Design and the founding Collection, 1837-51», Journal of the History of Collections 14, no. 1 (2002): 3-23, y «The making of South Kensington Museum II. Collecting Modern manufactures: 1851 and The Great Exhibition», Journal of the History of Collections 14, no. 1 (2002): 25-44.
} 
enseñanza de las llamadas artes mayores y las artes aplicadas o industriales, defendidas éstas por los más reformistas y por industriales para promover una instrucción muy dirigida a artífices y artesanos, como señala Prina. ${ }^{18}$

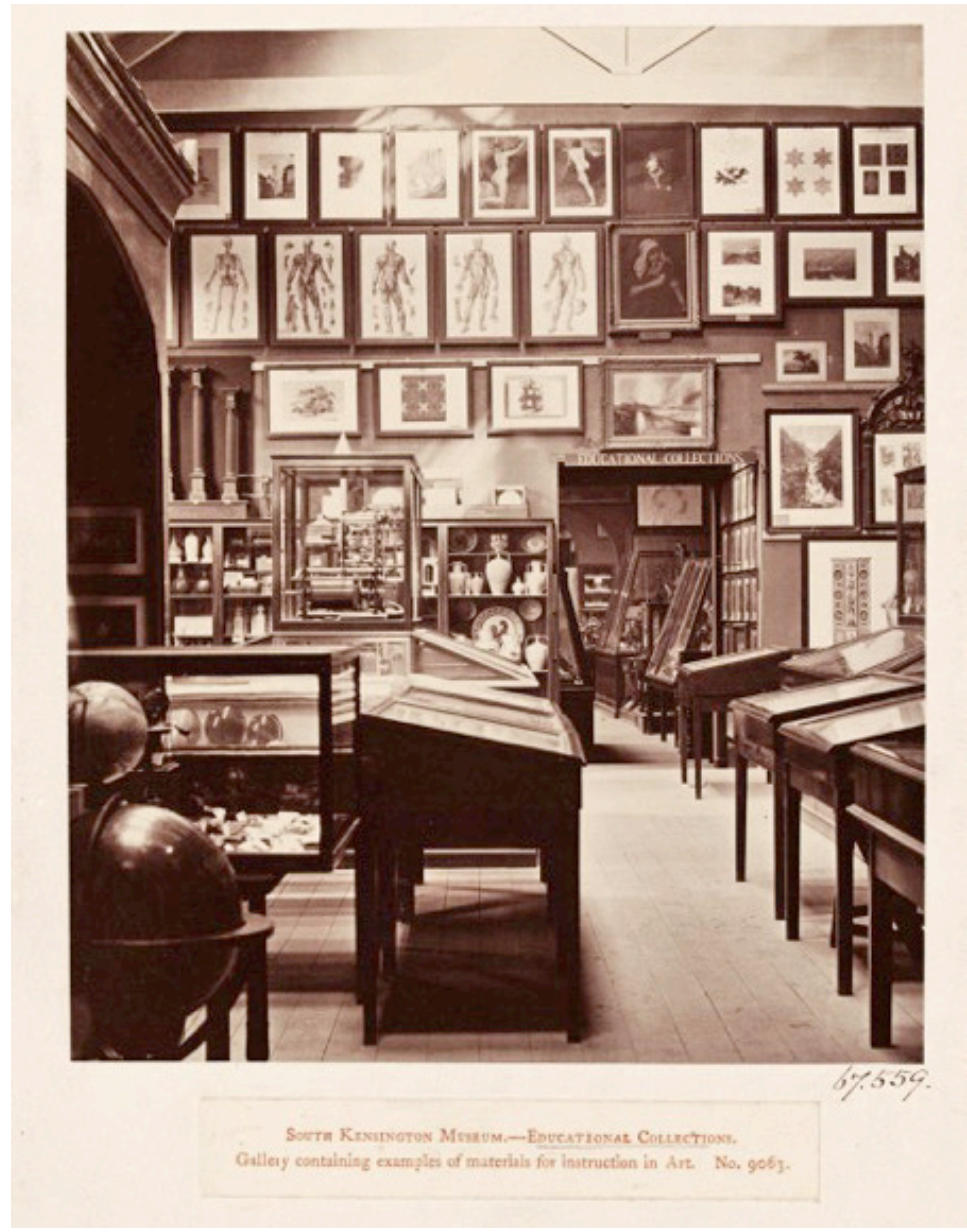

Figura 2. South Kensington Museum. Colecciones educativas. Galería que muestra ejemplos de materiales para la enseñanza del Arte. Inv. 67559, 1868-1869,

(C) Victoria and Albert Museum, London.

\footnotetext{
18 Daniela Prina, «Belgian decorative arts in the later nineteenth century. Needs for a National Museum and debates surrounding didactic collections in Brussels», Journal of the History of Collections 24, no 2 (2012): 257-274; John Turpin, «The South Kensington System and the Dublin Metropolitan School of Art 1877-1900», Dublin Historical Record 36, no. 2 (1983): 42-64. Publicado por Old Dublin Society, Stable URL: http://www.jstor.org/stable/30100731 (última consulta 20-08-2018).
} 
Al modo del SKM, surgían en los años setenta en Europa instituciones que tenían su origen en Exposiciones Universales, en un principio de carácter industrial y con claros planes pedagógicos. Podemos decir que los museos constituyen la prolongación permanente de dichas exposiciones, con cuyos objetos abrieron en muchos casos sus puertas. Viena inauguró en 1872 la Exposición permanente de material de enseñanza (Permanente Lechr-mittelausstellung), un museo escolar para que industriales y maestros dieran a conocer sus objetos. Al año siguiente la Exposición Internacional de Viena (1873) inauguraba con el lema «Cultura y Educación» y los dos museos más importantes de Suiza fueron creados como consecuencia de esta muestra. Zurich lo hacía en 1875 con una sección pedagógica bajo el nombre de Exposition scolaire permanente Schulanstellung, dependiente del museo industrial (Gewerbe Museum). Allí permaneció hasta 1882 que se organizó como institución independiente con el título de Pestalozzianum, y Berna abría un museo pedagógico en 1878. ${ }^{19}$ Todos ellos interesaron a Manuel Bartolomé Cossío en diversos viajes, algunos con Giner de los Ríos. Y en concreto en 1880, antes de volver a Madrid tras su estancia en Bolonia, visitó en Zurich el museo pedagógico. ${ }^{20}$

\section{EL MUSEO DE INSTRUCCIÓN PRIMARIA / MUSEO PEDAGÓGICO NACIONAL Y EL IMPULSO DE LA ILE A LA HISTORIA DEL ARTE Y LAS ARTES INDUSTRIALES}

En este contexto, y una década después del decreto de fundación del Museo Industrial, se inauguraba el Museo de Instrucción Primaria (6/05/1882) (después MPN),21 bajo la gestión de Juan Facundo Riaño (1829-1901), entonces director general de Bellas Artes (1881-1883). El MPN se alineaba así con la proliferación de este tipo de instituciones en Europa y EEUU. Se constituyó como un centro necesario con la función primordial de servir para la formación del magisterio, ${ }^{22}$ orientado éste en dos direcciones: formación de alumnos de diversa graduación y

\footnotetext{
19 García del Dujo, «El museo pedagógico nacional»,173-176.

20 Otero, «Los viajes de formación de Cossío entre 1879 y 1889», 169-179.

21 Javier Portus y Jesusa Vega, Cossío, Lafuente, Gaya Nuño. El descubrimiento del arte español. Tres apasionados maestros (Madrid: Nivola, 2004), 33-36.

22 Manuel B. Cossío, «El Museo Pedagógico de Madrid», en Museo Pedagógico de Instrucción Primaria, Documentos para su historia. Legislación. Organización. Memoria de sus trabajos (Madrid: Fortanet, 1886), 21.
} 
formación de maestros, ${ }^{23}$ con un planteamiento más propio de un centro de investigación y enseñanza que de un depósito de colecciones, o lo que se entendía por una institución museística. Consideraba relevante la cuestión material, en el sentido de estimular la fabricación a nivel nacional de material escolar, así como recabar del mundo industrial series pedagógicas susceptibles de utilización en la enseñanza. ${ }^{24}$ Este segundo aspecto le acercaba a una tarea propia de los museos industriales que, como hemos visto, eran algo anteriores a estos. ${ }^{25}$ En definitiva, se podría explicar como un laboratorio de investigación educativa, debido a la inspiración de Giner de los Ríos, y en línea con los proyectos educativos y pedagógicos europeos. ${ }^{26}$ Como señala Javier Mateo, esto era lo que marcaba el hecho diferencial de esta institución, el objetivo intrínseco de la practicidad enfocada a la enseñanza, en un contexto marcado por la controversia entre los que se decantaban por la valoración artística de los objetos, y los que ponían en primer lugar su condición de objetos cotidianos, sin olvidar el sentido de la estética de los mismos. ${ }^{27}$

¿Cómo casar todo esto con la labor desempeñada por la ILE? Varios miembros de esta institución fueron decididamente proclives a la enseñanza de la Historia del Arte y en sus publicaciones se encuentra, incluso en las actividades que emprendían, un claro apoyo a esta disciplina y, en el terreno que nos ocupa, a las artes aplicadas e industriales. Así, Hermenegildo Giner de los Ríos (1847-1923), uno de sus fundadores, escribió el primer manual de Historia del Arte: Teoría del Arte e Historia de las Bellas Artes en la Antigüedad (1873),,28 y el Manual de Estética y Teoría del Arte e Historia abreviada de las artes principales hasta el cristianismo, con especial atención a las artes subordinadas e industriales, y esto le situaba, en una

\footnotetext{
${ }^{23}$ Museo Pedagógico de Instrucción Primaria, Catálogo Provisional (Madrid: Imprenta de Fortanet, 1890).

${ }^{24}$ Museo Pedagógico de Instrucción Primaria, Catalogo provisional, XIX.

${ }_{25}$ María Bolaños, Historia de los museos en España. Memoria, cultura y sociedad (Gijón: Trea, 1997), 261-267.

26 Ángel García del Dujo, «Manuel B. Cossío y el Museo Pedagógico Nacional», Bordón. Revista de Pedagogía 258 (1985): 367-384; Natividad Ortega Morales, La enseñanza-aprendizaje del arte. Una innovación educativa de la institución Libre de Enseñanza (Granada: Grupo Editorial Universitario, 2002), 169-187.

27 Javier Mateo de Castro, «El museo: un espacio educativo para la igualdad social. El caso pionero del Museo Pedagógico Nacional en España (1882-1941)», El futuro del pasado 8 (2017): 112.

${ }^{28}$ Hermenegildo Giner de los Ríos, Teoría del Arte e Historia de las Bellas Artes en la Antigüedad (Madrid: Baeza, 1873).
} 
corriente de pensamiento cercana a los movimientos de Arts and Crafts y Art Nouveau. ${ }^{29}$ Pero la trascendencia de las iniciativas de la ILE en el ámbito oficial fue escasa, y lo mismo ocurrió respecto a la historia del arte que, aunque figuró como disciplina en algunos planes de estudio de Bachillerato, donde realmente tuvo proyección fue en los principios, iniciativas y actuaciones de la ILE. ${ }^{30}$ La influencia del krausismo fue también determinante en este sentido. ${ }^{31}$

Otras dos personalidades, Francisco Giner de los Ríos (1839-1915) y Juan Facundo Riaño (1829-1901) escribieron sendas obras dedicadas al tema de las artes industriales. Riaño era historiador del arte y una persona muy cercana a Giner de los Ríos, del que fue su mentor y figura esencial en la valoración social de la historia del Arte. ${ }^{32}$ En su casa se gestó el amor a la Historia del Arte con todo su sentido pedagógico. ${ }^{33}$ Por otra parte, Riaño estaba muy vinculado al mundo anglosajón familiarmente. Estaba casado con Emilia Gayangos Redwell, hija del notable arabista Pascual Gayangos. Su trabajo fue muy importante como asesor en el South Kensington Museum, pues recomendó las adquisiciones de piezas españolas de joyería, tejidos, cerámica o vidrio para la colección del museo. ${ }^{34}$ Basándose en ellas publicó The industrial Arts in Spain (1879), ${ }^{35}$ primera visión de conjunto sobre este tema.

\footnotetext{
29 María Rosario Caballero, «El primer Manual de Historia del Arte con destino al Bachillerato. Su autor: Hermenegildo Giner de los Ríos», Imafronte 15 (2000-2001): 23. http://revistas.um.es/imafronte (última consulta 6-08-2018).

${ }^{30}$ María Rosario Caballero, «La Historia del Arte en la enseñanza secundaria con destino al Bachillerato, Perspectiva histórica y posibilidades de futuro», Imafronte 8 y 9 (1992-93): 52-56, https:// dialnet.unirioja.es/servlet/articulo?codigo=233380 (última consulta 1-08-2018); Inicios de la historia del arte en España: la Institución Libre de Enseñanza (1876-1936) (Madrid: CSIC, Instituto de Historia, Departamento de Historia del Arte, 2002).

${ }^{31}$ Leticia Sánchez de Andrés, «Manuales de origen krausista para la enseñanza de la estética y la historia del arte y de la música en los manuales de bachillerato», Arbor. Ciencia, pensamiento y cultura 187, no. 749 (2011): 535-545; Ortega, La enseñanza-aprendizaje del arte, 17-42 y 100-110; Caballero, «El primer Manual de Historia del Arte», 17-28.

32 http://www.rah.es/juan-facundo-riano-montero/, última consulta 1-09-2018.

${ }_{33}$ Manuel B. Cossío, El Greco (Madrid: Espasa Calpe, 1983): 23.

${ }^{34}$ Marjorie Trusted, «In all cases of difference adopt Signor Riaño’s view. Collecting Spanish decorative arts at South Kensington Museum in the late nineteenth century", Journal of the History of Collections 18, no. 2 (2006): 228-236. http://jhc.oxfordjournals.org/content/18/2/225.full (última consulta 1-09-2018).

35 Juan Facundo Riaño, The industrial arts in Spain (London: Chapman and Hall, 1890).
} 
Giner de los Ríos, fundador y figura fundamental de la ILE, escribió un texto sobre Estudios de Artes Industriales ${ }^{36}$ en el que defendía la importancia de los objetos decorativos como portadores de conocimiento y significado en relación con el contexto histórico al que se vinculaban, y hacía esta reflexión sobre el conocimiento en profundidad de este tipo de piezas:

considerar que hay dentro de aquel barro, de aquellas formas, de aquella ornamentación; el íntimo enlace que guardan todos sus elementos con las costumbres, el género de vida, el medio natural, los gustos, las influencias, el espíritu entero de un pueblo o un tipo de cultura es cosa que pide otra atención más detenida y otra manera de mirar el cacharro. [...] En esta indomable solidaridad de todos los factores de un ciclo, un jarrón del Retiro habla muy bajito, pero muy claro, de la Revolución francesa, y una silla pseudo-gótica del año 20, de Restauración y Santa Alianza [...] Todo está en todo. ${ }^{37}$

Se anticipaba así a un modo de valoración de los objetos muy en boga en nuestro tiempo, que se pone de manifiesto en diferentes formatos: publicaciones, exposiciones, curso o simposios, entre otros. ${ }^{38}$

Giner fue sucedido por Manuel B. Cossío (1857-1935), al frente de la ILE. Pertenecía al grupo de profesores que se habían formado ya en su seno y que se incorporaron como docentes a la Institución a partir de 1881.39 Ambos, como muchos de los institucionistas, mostraban su atracción por

\footnotetext{
${ }^{36}$ Francisco Giner, Estudios sobre artes industriales (Madrid: Librería de José Jorro, 1892).

${ }^{37}$ Francisco Giner, Estudios sobre artes industriales y Cartas literarias, en Obras Completas de Francisco Giner de los Ríos, vol. XV (Madrid: Imprenta de Julio Cosano 1926), 3-4.

${ }_{38}$ Hispanic Society of America (Nueva York), Mitchell A. Codding, María Dolores Jiménez-Blanco, Patrick Lenaghan, \& Museo Nacional del Prado, Tesoros de la Hispanic Society of America : Visiones del mundo hispánico (Madrid: Museo Nacional del Prado, Nueva York: Hispanic Society of America, 2017); Manuel Lucena Giraldo, 82 objetos que cuentan un país: Una historia de España (Barcelona: Taurus, 2015); Neil MacGregor, La historia del mundo en 100 objetos (Barcelona: Debate, 2012); Joan Santacana i Mestre y Naira Llonch Molina, Manual de didáctica del objeto en el museo (Gijón: Ediciones Trea, 2012); Silvia Canalda y Albert Martí, Los objetos hablan. Dossier para profesores (Madrid: Museo Nacional del Prado, Obra Social La Caixa, 2009); Ángela García Blanco, Didáctica del museo: El descubrimiento de los objetos (Madrid: Ediciones de la Torre, 1994).

${ }^{39}$ Fundación Francisco Giner de los Ríos (Institución Libre de Enseñanza), http://www.fundacionginer.org/historia.htm, (última consulta 3-08-2018).
} 
Inglaterra como referencia estética. ${ }^{40}$ Cossío, que coincidía con Riaño en el interés por las artes industriales y populares, escribió un artículo en el que se refería al sentido de la estética en las casas inglesas y a las creaciones de Morris y Benson. ${ }^{41}$ No en vano, las teorías de Morris reivindicaban la unión de utilidad y belleza que confluía en los objetos cotidianos, y la sensibilización frente a esta realidad era uno de los objetivos de la misión del MPN, en el sentido de transmitir, mediante la educación, la importancia de lo consuetudinario, lo popular y el trabajo bien hecho.

Asimismo, no conviene olvidar que la relación entre museos industriales y museos pedagógicos es un hecho, pues se trataba de dos tipos de instituciones cuyo objetivo era contribuir al progreso y mejora de la sociedad, a la extensión de la cultura y el saber en ámbitos sociales más amplios. Esto les acreditaba como necesarios desde el punto de vista educativo y les dio carta de naturaleza como instituciones al servicio del país y de su contribución hacia el futuro, así como difusores de la historia y la memoria de las naciones.

Al tiempo, la preocupación por la enseñanza del arte era un hecho tanto para los historiadores como para los críticos de arte. Desde Madrid y Barcelona se venía defendiendo, hacía tiempo, la necesidad de un museo industrial, así como de la organización de exposiciones de arte decorativo o centradas en los llamados bellos oficios, ${ }^{42}$ que permitirían confrontar los logros de los artífices y talleres con las necesidades de la sociedad y ver la relación con las tendencias artísticas del momento. Ya en 1892, el ingeniero, político, historiador y arqueólogo, entre otras muchas cosas, Pablo Alzola y Minondo,43 en su obra El arte industrial en España (1892), detectaba un vacío y reivindicaba la necesidad de un museo central industrial en nuestro país. Y es significativo y curioso que

\footnotetext{
40 Manuel Bartolomé Cossío, «Sobre la educación estética», Boletín de la Institución Libre de Enseñanza XI, no. 241 (1887): 321-322.

${ }^{41}$ Manuel Bartolomé Cossío, «Más sobre la educación estética en Inglaterra», Boletín de la Institución Libre de Enseñanza XI, no. 241 (1887): 321-322 y 353-354.

${ }_{42}$ Lola Caparros Masegosa, «La institucionalización de las artes decorativas en España: de sección en las Exposiciones Nacionales de Bellas Artes a Exposición Nacional de Artes Decorativas (18971910)», Res Mobilis. Revista internacional de investigación en mobiliario y objetos decorativos 5, no. 5 (2016): 75-100. https://dialnet.unirioja.es/descarga/articulo/5290511.pdf (última consulta 6-07-2018).

${ }_{43}$ Sobre Alzola véase Javier González de Durana, Puente de Hierro para la Ría de Bilbao de D. Pablo Alzola (Bilbao: Colegio Oficial de Aparejadores y Arquitectos Técnicos de Vizcaya, 1986).
} 
señalase en su repaso de los museos españoles, en primer lugar al Museo de Instrucción Primaria, aludiendo a sus colecciones de objetos industriales y labores procedentes de valiosos donativos de particulares "porque este recurso será indispensable para la creación de los de arte industrial».44 Alzola reclamaba en sus conclusiones energía para reorganizar la enseñanza y la creación de un museo de esta tipología, por lo que le podemos considerar un precedente de la actitud y la apuesta del primer director del Museo Nacional de Artes Industriales: Rafael Doménech. ${ }^{45}$

También a nivel institucional, como recuerda Francisco Alcántara, la Academia de Bellas Artes, al terminar el curso en 1908, presentaba un documento relativo a la política artística del que extractamos lo siguiente: "Los pueblos que aspiran a tener la hegemonía del mundo, comienzan educando artísticamente a sus obreros para que los productos de su comercio y de su industria atraigan por su aspecto exterior, tanto como por sus condiciones utilitarias». ${ }^{46}$ Vinculaba así, entre otras cosas, la atención a las artes aplicadas al progreso del país, sin que hubiera cambiado en demasía lo que se proponía ya a mediados del siglo anterior en relación con la creación de un museo industrial.

\section{HACIA LA FUNDACIÓN DEL ACTUAL MUSEO NACIONAL DE ARTES DECORATIVAS}

Curiosamente en el contexto en el que nos movemos, en torno a las décadas de 1870-1880, es cuando nacía el que sería, a comienzos del siglo XX, el primer director del Museo Nacional de Artes Industriales (en adelante MNAI), Rafael Doménech Gallisá (1874-1929). Fue jurista, pero su vida se centró en torno a las artes como crítico, historiador, docente y director de museo. Merece señalarse alguno de sus escritos, ${ }^{47}$ donde

\footnotetext{
${ }_{44}$ Pablo Alzola Minondo, El arte industrial en España (Bilbao: Casa de la Misericordia, 1892), 263. http://bvpb.mcu.es/es/consulta/registro.cmd?id=411560 (última consulta 6-07-2018). Existe una edición de esta obra, del Colegio de Ingenieros de Caminos, Canales y Puertos, con prólogo de Sofía Rodríguez Bernis.

${ }^{45}$ Alzola, El arte industrial en España, 266-271.

${ }^{46}$ Francisco Alcántara, «Política artística», El Imparcial XLII, no. 14884 (21 de agosto de 1908): 3.

${ }^{47}$ Rafael Doménech, «La enseñanza superior de las industrias artísticas en Valencia», Revista Contemporánea XXVI, no. CXIX (30 de julio de 1900): 122-131, y «La enseñanza superior de las industrias artísticas en Valencia", Revista Contemporánea XXVI, no. CXIX (15 de agosto de 1900): 225-244.
} 
reflexionaba sobre la enseñanza en relación con las artes industriales. También para él eran referentes países como Inglaterra, Francia o Bélgica. Igual que los miembros de la ILE, miraba al mundo anglosajón y a sus innovaciones debidas a Morris, a Walter Crane, etc., y al progreso conseguido en menos de medio siglo. Propuso, entre otras muchas cosas, fusionar las Escuelas de Bellas Artes y las de Artes y Oficios en las Escuelas Superiores de Artes e Industrias, y que éstas dieran cabida a enseñanzas propias de industrias locales, lo que mejoraría la formación de obreros, artífices e industriales. ${ }^{48}$

En su opinión, había que cambiar el sistema de enseñanza, idea que ya proponía unos años antes. Doménech defendía incluso un sistema aplicado a las necesidades y rasgos propios de cada ciudad; es decir, entendía que las industrias de Bilbao eran «técnicas y científicas» y las de Valencia, eran "preferentemente artísticas». Para él, una reforma en la enseñanza de las artes decorativas e industriales realmente eficiente y operativa, pasaba por un cambio radical en favor de la necesaria formación práctica de los alumnos. El progreso requería apartarse de la vinculación con el pasado y su imitación, poner fin a los historicismos y tomar la naturaleza como principal fuente de inspiración, en lugar de utilizar el pasado para reproducirlo o copiarlo. ${ }^{49}$

Es significativo, por otra parte, que sus escritos los encontramos en publicaciones de ideología vinculada a la Institución Libre de enseñanza, así los textos anteriores en La Lectura, "revista de ciencias y artes», 50 dirigida por Francisco Acebal, que fue nombrado, en 1907, vicesecretario de la Junta para la Ampliación de Estudios e Investigaciones Científicas, y en la Revista Contemporánea (1875-1907). ${ }^{51}$

Doménech fue la persona que desde sus escritos concretó más en relación con la creación de un nuevo museo de artes industriales, siendo

\footnotetext{
48 Doménech, «La enseñanza superior de las industrias artísticas en Valencia», Revista Contemporánea, (30 de julio de 1900): 122-131.

${ }^{49}$ Rafael Doménech, «Exposición Nacional de Bellas Artes de 1906», La Lectura. Madrid VI, no. 69 (1906): 22-24.

${ }^{50} \mathrm{http}: / /$ hemerotecadigital.bne.es/details.vm?q=id:0002631544\&lang=en (última consulta 17-072018).

${ }^{51} \mathrm{http}: / /$ hemerotecadigital.bne.es/details.vm?q=id:0002283020\&lang=es (última consulta 17-072018).
} 
partícipe asimismo en un debate sobre las artes decorativas y su importancia para el progreso de nuestro país, existente en España en aquellos años. ${ }^{52}$ El texto de la Revista Contemporánea de 1900, suponía casi el bosquejo de un plan museológico o la base del proyecto de creación de una futura institución museística. Muy sintéticamente diremos que lo planteaba como un medio de educación artística para el público, un recurso de exhibición de las industrias artísticas, no sólo de índole local o regional, sino que parecía apuntar a la idea de un museo nacional. Se planteaba incluso la procedencia de las piezas que constituirían los fondos de la colección, basándose en ideas que en Europa ya se venían poniendo en práctica desde el siglo pasado y de las que, de forma contrastada, teníamos noticias en nuestro país. ${ }^{53}$

Y como conclusión a sus reflexiones y condiciones, añadía algo que le parecía singularmente importante y que recuerda mucho a los principios fundacionales del MPN: "Convertir el Museo, no en un almacén de obras artísticas — como son los de España—, sino en un organismo lleno de vida; que no sea un lugar de puro ornato donde se vaya sólo a ver, sino que sea un sitio de verdadera cultura». ${ }^{54}$ Un espacio en el fuesen habituales las conferencias abiertas al público en general y la divulgación de las colecciones del museo en pequeños estudios y folletos, y un centro que diera todo tipo de facilidades a los fabricantes para inspirarse en los objetos allí custodiados. ${ }^{55}$ Lo que nos indica que estaba muy cerca de las ideas de los reformistas europeos en estas materias.

De manera activa trabajó, escribió, defendió y reivindicó un cambio en torno a las artes aplicadas, ${ }^{56}$ por lo que fue considerado la persona idónea para asumir la dirección del Museo Nacional de Artes Industriales, creado por Real Decreto de 30 de diciembre de 1912. La publicación

\footnotetext{
${ }^{52}$ Fernando García Rodríguez, «Teoría del arte: las artes decorativas en la prensa española del primer tercio de siglo», en Historiografía del Arte Español en los siglos XIX y XX, ed. CSIC (Madrid: Alpuerto, 1995), 450-470.

53 Doménech, «La enseñanza superior de las industrias artísticas en Valencia», 243; María Villalba Salvador, «Rafael Doménech y el Museo Nacional de Artes Decorativas. Génesis de una colección», además de. Revista on line de artes decorativas y diseño 1 (2015): 46-48, http://www.ademasderevista. com/pdfs/numero1/articulo_villalba.pdf (última consulta 2-09-2018).

${ }^{54}$ Doménech, «La enseñanza superior de las industrias artísticas en Valencia», 244.

${ }_{55}$ Doménech, «La enseñanza superior de las industrias artísticas en Valencia», 244.

56 Villalba, «Rafael Doménech y el Museo Nacional de Artes Decorativas», 46-50.
} 
de su Reglamento, desarrollo del decreto de fundación, se producía un año después ${ }^{57}$ y en el artículo $1^{\circ}$ ya se destacaba la necesidad de poner al alcance del público el conocimiento de la técnica, del trabajo manual y de la teoría artística, así como la historia de las artes industriales a través de obras únicas, españolas y extranjeras, desde el arte antiguo al arte contemporáneo. Su función esencial no sería «la mera delectación artística y contemplativa, sino el promover la cultura artística y técnica de las artes aplicadas en el público y especialmente en los artistas, industriales y obreros» $\left(\right.$ art. $\left.1^{\circ}\right) .58$

El Museo se definía como un organismo de educación teórico práctica, no una simple exposición de objetos, por lo que «la Dirección facilitaría el estudio de los objetos de sus colecciones, autorizando las copias gráficas, los exámenes y mediciones directas, y cuantas informaciones soliciten los visitantes» (art. 12). ${ }^{59}$ La adquisición de objetos antiguos se guiaba por el hecho de que fuese portador de una enseñanza propia de las artes decorativas o de su evolución, o de un procedimiento técnico, no por su valor arqueológico. Lo mismo ocurría con las piezas contemporáneas, que cerraban el círculo de la evolución histórica. La idea era formar series técnicas y series históricas. ${ }^{60}$

\section{PARALELISMOS ENTRE EL MUSEO NACIONAL DE ARTES INDUSTRIALES Y EL MUSEO PEDAGÓGICO NACIONAL}

En todo el proceso de la puesta en funcionamiento del MNAI, en sus principios y maneras de proceder, descubrimos grandes coincidencias con los planteamientos del Museo Pedagógico Nacional de Cossío. Este se planteaba como un centro de investigación y enseñanza que serviría

\footnotetext{
57 «Real Decreto de 30 de diciembre de 1912», Boletín Oficial del Ministerio de Instrucción Pública y Bellas Artes 2 (7 de enero de 1913).

58 «Reglamento. Capítulo primero. De la finalidad del Museo. Art. $1^{\circ}$ » Boletín Oficial de Ministerio de Instrucción Pública y Bellas Artes V, no.7 (23-01-1914): 3.

59 «Reglamento. Capítulo primero. De la finalidad del Museo. Art. $1^{\circ}$ », Boletín Oficial de Ministerio de Instrucción Pública y Bellas Artes V, no. 7 (23-01-1914): 3.

60 Sobre los inicios del MNAI, véase Ana Cabrera Lafuente, «El Museo Nacional de Artes Decorativas en sus primeros años", además de. Revista on line de artes decorativas y diseño 1 (2015): 92-96, http:// www.ademasderevista.com/pdfs/numero1/articulo_cabrera.pdf (última consulta 2-09-2018; María Villalba Salvador y Ana Cabrera Lafuente, «El Museo Nacional de Artes Industriales, hoy Museo Nacional de Artes Decorativas (Madrid) (1912-1927)», Revista de Museología. HdM 36 (2006): $117-123$.
} 
a la formación y cultura del Magisterio, y el MNAI contribuiría a la formación de profesionales, artistas, artífices o artesanos y, de hecho, también a profesores y alumnos de la Escuela de Magisterio.

\section{La biblioteca}

En este sentido, la biblioteca era esencial en ambas sedes. Lafuente Ferrari recuerda que la del MPN era una «creación de D. Manuel Bartolomé Cossío que recogía y exhibía en sus salas material escolar moderno para orientación de las escuelas, maestros e inspectores... El Museo se completaba con una biblioteca pública literalmente abierta a los lectores y atendida por profesores y discípulos del Sr. Cossío». ${ }^{61}$

En el MNAI la biblioteca estaba abierta a artistas, artífices, profesores de Escuelas de Artes y Oficios, e investigadores. Por allí pasaron, en lo que más interesa a este trabajo, numerosas maestras y, sobre todo alumnas de la Escuela Normal Central, centro importante para la formación de la mujer en el primer tercio del siglo XX. ${ }^{62}$ Todo ello era acorde con el Plan de Estudios de las Escuelas Normales de 1914, que responde al Real Decreto de 30 de agosto de ese año y que estuvo vigente hasta 1931. En él se contemplaba para Primer curso: Costura (para las maestras), Segundo Curso: Bordado y Corte, y Tercer Curso: Corte y Labores. ${ }^{63}$ Quizás los años más significativos fueron los transcurridos de 1919 a 1922, tal como evidencian las solicitudes realizadas para consultas, copia y documentación en el museo. En enero y febrero de 1919 acudieron seis maestras nacionales de Madrid y Toledo (su consulta fue en relación con una antología del ornamento); y setenta y dos alumnas de la Escuela Normal Central de Maestras. Las solicitudes encontradas en el archivo del MNAD dan cuenta del interés de estas alumnas por copiar dibujos y motivos de bordados antiguos españoles. En algunos casos queda reseñado en la ficha de solicitud (la primera 19/04/19). ${ }^{64}$ Del año

\footnotetext{
${ }^{61}$ Portús y Vega, El descubrimiento del arte español, 38 (Recogido de Lafuente, «Memorias» (inéditas)).

${ }_{62}$ Isabel Rodríguez Marco y Ana Cabrera Lafuente, «The Involvement of Women in the National Museum of Decorative Arts of Madrid (Spain): 1912-1942», Museum History Journal II, no. 2 (2018): 153-173. DOI: 10.1080/19369816.2018.1529221.

${ }^{63}$ Agustín Escolano Benito, «Las Escuelas Normales. Siglo y medio de perspectiva histórica», Revista de Educación 269 (1982): 55-76.

64 «Peticiones de usuario», Archivo del MNAD, C.0002, D.03.
} 
1920 hay cuatro demandas para copiar objetos del museo en bordados, o dibujos de labores, ${ }^{65}$ y veintidós en el año 1921 para copiar dibujos de malla y deshilados, y motivos en bordados. ${ }^{66}$ También lo solicitan alumnas de la Escuela Superior de Magisterio (1919, 1921, 1922)67 y maestras nacionales (1922) para trabajar con dibujos de labores. Existe una instancia solicitando un certificado a la dirección del museo sobre su formación en el mismo sobre sus estudios de labores, bordados del siglo XVI, y luego los estudios dirigidos de sus alumnos. ${ }^{68}$ Las peticiones continúan en 1923 en el mismo sentido o en relación con el aprendizaje del arte del batik, ${ }^{69}$ consultas en la biblioteca de libros sobre historia general del arte $(1924,1926),{ }^{70}$ sobre estudios de indumentaria y encajes (1930), ${ }^{71}$ y algunas más de copias de objetos del museo (1930 y 1931).72 Todo esto nos habla de un trabajo riguroso, que va a las fuentes originales y que muestra la relación entre el museo y la formación de maestras.

\section{La Junta para Ampliación de Estudios e Investigaciones Científicas}

Hay otros paralelismos, como corrobora el hecho de que desde el inicio fuera decisiva en las dos instituciones la relación con la Junta para Ampliación de Estudios e Investigaciones Científicas (JAE), organismo autónomo dependiente del Ministerio de Instrucción Pública y muy influido por el pensamiento de Francisco Giner de los Ríos y la Institución Libre de Enseñanza. ${ }^{73}$

\footnotetext{
65 "Peticiones de usuario», Archivo del MNAD, C.0002, D.10.

66 «Peticiones de usuario», Archivo del MNAD, C.0002, D.12.

67 «Peticiones de usuario», Archivo del MNAD, C.0002, D.15.

68 Solicitud de certificado de María Castellanos y Díaz, dirigida al director del MNAI, de 25-08-1922, en «Peticiones de usuario», Archivo del MNAD, C.0002, D.15.

69 «Peticiones de usuario», Archivo del MNAD, C.0004, D.11.

${ }^{70}$ Así aparece citado en la ficha de solicitud. Posiblemente se refiere a la obra Historia del arte en todos los tiempos y pueblos de Kark Woermann, publicada por Saturnino Calleja en seis volúmenes en 1923-1925. "Peticiones de usuario», Archivo del MNAD, C.0004, D.17, y C.0004, D.32.

71 «Peticiones de usuario, Archivo del MNAD, C.0007, D.19.

72 «Peticiones de usuario», Archivo del MNAD, C.0007, D.19 y C.0007, D.47.

${ }^{73}$ Memoria correspondiente al año 1907. Junta de Ampliación de Estudios e Investigaciones Científicas (Madrid, 1908), 18. http://cedros.residencia.csic.es/imagenes/Portal/ArchivoJAE/memorias/001.pdf (última consulta 2-09-2018).
} 
Desde el museo dirigido por Cossío se colaboraba en la preparación pedagógica de excursiones de maestros al extranjero mediante las ayudas de dicha Junta. El propio Cossío viajó por el centro y norte de Europa becado por la JAE en 1909 y 1910, con lo que se empapó de los planteamientos educativos, además del conocimiento del patrimonio histórico artístico de países como Dinamarca, Suecia Noruega Alemania, Suiza y Bélgica. ${ }^{74}$ De otra parte, desde el MNAI su director, subdirector y otros colaboradores recibieron becas de este mismo organismo para conocer los principales museos industriales europeos. En ambos casos se trataba de conseguir la puesta al día en relación con Europa.

Hubo tres personas vinculadas al MNAI cuya formación museológica debe mucho a las estas ayudas. La JAE había considerado de enorme importancia la formación de los investigadores, profesionales y estudiosos de las artes industriales. De las ciento catorce ayudas concedidas a la materia de arte, cincuenta y cuatro se destinaron a este ámbito de las artes. ${ }^{75}$ En especial al conocimiento de las colecciones de artes aplicadas e industriales de los museos europeos. Los beneficiarios fueron Rafael Doménech, ya entonces director del Museo Nacional de Artes Industriales, Luis Pérez Bueno (1885-1954) y Francisco Pérez Dolz (1887-1958). ${ }^{76}$ Estos últimos eran docentes vinculados a las Escuelas de Artes y Oficios de Madrid y Granada, respectivamente. Fue especialmente relevante el viaje realizado en 1914 al Pabellón Marsán del Louvre, al Victoria \& Albert Museum y a su filial, el Bethnal Green Museum, creado en una barriada popular y con fines claramente educacionales, y a Bruselas donde visitaron el Museo del Cincuentenario. El viaje tuvo que cancelarse a medio camino debido al estallido de la Gran Guerra. La pensión se concedió con el objetivo de «conocer el funcionamiento y la organización de los Museos de Artes Industriales y de carácter popular; comités para el progreso de éste; organización y funcionamiento de la enseñanza y

\footnotetext{
74 Portus y Vega, El descubrimiento del arte español, 28.

${ }_{75}$ Natacha Seseña, «Los becarios de arte de la Junta para Ampliación de Estudios», en 1907-1987. La Junta para Ampliación de Estudios e Investigaciones Científicas 80 años después, coord. José Manuel Sánchez Ron (Madrid: CSIC, 1989): 557-585, vol. II. http://archivojae.edaddeplata.org/jae_app/ (última consulta 2-09-2018).

76 Memoria correspondiente al año 1914 y 1915. Junta de Ampliación de Estudios e Investigaciones Cientificas (Madrid, 1916), 55-57 y 93. http://cedros.residencia.csic.es/imagenes/Portal/ArchivoJAE/ memorias/005.pdf (última consulta 3-09-2018).
} 
laboratorios existentes en los mismos Museos». ${ }^{77}$ Esto indica que en España, existía una sensibilidad enmarcada en la recuperación del mundo popular y el interés por la intrahistoria que se generó a finales de siglo. ${ }^{78}$ Con la perspectiva del tiempo pasado y contemplando la colección del museo, todo adquiere sentido.

La guerra había truncado la continuación del viaje previsto: Holanda, Alemania, Austria-Hungría y Suecia. Pero, si a lo que visitaron sacaron tanto partido, qué no hubiera sido de poder recorrer los museos restantes. Por otra parte, la formación de estos pensionados continuó después de la guerra, desde 1922 a 1934. Pérez Bueno estudiaría las colecciones españolas en los museos europeos, y Pérez Dolz se formó sobre todo en cuestiones prácticas, en lo relativo a la organización de la enseñanza de la mujer, y en el montaje de exposiciones, lo que repercutiría en la didáctica posterior en el MNAI. ${ }^{79} \mathrm{Su}$ aprendizaje fue reconocido por Doménech, que fue quien lo requirió para trabajar en el Museo. ${ }^{80}$ No son los únicos testimonios encontrados. También algunas fichas hablan de ello, como la correspondiente al número de inventario 97, del fondo antiguo: «Objeto: Un almohadón. Malla bordada [...] Proyecto del Profesor Sr. Pérez Dolz ejecutado con destino al fondo de enseñanza práctica de este museo». Incluso numerosos montajes de tejidos antiguos, por ejemplo, aquellos en los que se desgrana su desarrollo decorativo, como es el caso de un conjunto de encajes cuyo montaje muestra la datación cronológica y la técnica utilizada (Inv. CE7088) (11 $^{2}$ (figura, 3).

\footnotetext{
77 Memoria correspondiente al año 1914 y 1915. Junta de Ampliación de Estudios e Investigaciones Científicas (Madrid, 1916): 55 y 93.

${ }_{78}$ Natacha Seseña, «La historiografía del arte popular en el siglo XX», en Historiografía del Arte español en los siglos XIX y XX, ed. CSIC (Madrid: Alpuerto, 1995), 449-457; Portus y Vega, El descubrimiento del arte español, 35; Sofía Rodríguez Bernis, «Cossío y las artes populares», en El arte de saber ver, 185-191.

79 Villalba, «Rafael Doménech y el Museo Nacional de Artes Decorativas», 46-50.

${ }^{80}$ Carlos González Spresati, «Las metamorfosis estéticas de Pérez Dolz», Boletín de la Sociedad Castellonense de Cultura V (1924): 254-255.

${ }^{81}$ Cabrera, «El Museo Nacional de Artes Decorativas en sus primeros años 1912-1930», 95.
} 
Nümero 97

objeto Mu a luohadun. Malla hordadaNharpora en el ceutno, elecucutos flora les cular aígulos-colores crevera Pragecto del praferar fo. Pener Nots

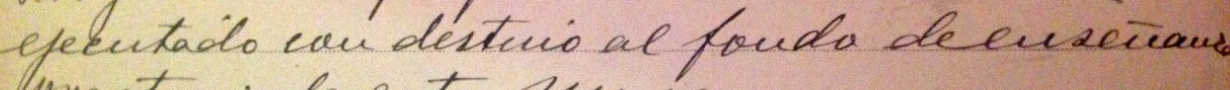
macteca de este nucseo

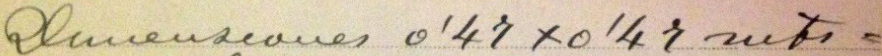
Goba - trilo - Malla bordacla =

Hdquísición

sala $\mathcal{J}^{\circ}=$

Figura 3. Ficha del fondo antiguo del Archivo del MNAD del Proyecto de enseñanza práctica del profesor Pérez Dolz. Fuente: elaboración propia.

Creemos, como dice Ana Cabrera, ${ }^{82}$ que se trata del primer museo en el que se creó algo equivalente al área de educación y acción cultural de los museos nacionales en la actualidad, lo cual es de enorme importancia, pues era acorde, además, con los planteamientos educativos más progresistas. ${ }^{83}$

\section{Una aproximación a los planteamientos concurrentes en los fondos de la colección el MPN y del MNAI}

Se puede afirmar, por tanto, que los principios que inspiraron ambos museos y muchos de sus proyectos son convergentes. Pero también es

\footnotetext{
82 Cabrera, «El Museo Nacional de Artes Decorativas en sus primeros años 1912-1930», 90-92.

83 Sofía Rodríguez Bernis y Paloma Muñoz Campos García, «El museo constructor de otros contextos. Cien años del Museo Nacional de las Artes Decorativas», Anales de Historia del Arte 24, no. especial (2014): 463.
} 
significativo que existan coincidencias en cuanto a determinadas tipologías de objetos. Así, el catálogo provisional del MPN, el único publicado, muestra una sección de arte industrial «para estimular la fabricación nacional de toda clase de material y mobiliario escolares, eligiendo los mejores sistemas aplicables en nuestro país», y "fomentar las colecciones del museo, solicitando en especial de comerciantes e industriales donativos de muestras y objetos para formar series pedagógicas (v.g. tecnología) que sirvan de modelo y estudio a los maestros». ${ }^{84}$

Y, si observamos en paralelo al MNAI, hemos visto que uno de los objetivos iniciales era la búsqueda de piezas que formasen series significativas relativas a la evolución histórica de las artes decorativas y el progreso técnico, con incidencia en su utilización como recurso pedagógico. Por ejemplo, en el viaje que realizaron Doménech y Pérez Bueno a Inglaterra en 1914, se adquirieron piezas contemporáneas que hablaban de cómo la cerámica española de reflejo metálico había influido en la creación de objetos de determinadas manufacturas como Pilkington, Doulton y Minton, piezas que se adquirieron en aquel viaje para la colección fundacional. Las fichas de catálogo del museo inicial así lo demuestran..$^{85}$ También se encuentran en el MNAD adquisiciones de tejidos de Morris que pudieran ser procedentes del taller de Merton Abbey o comprados en 1920 durante la Exposición de la Commonwealth, ${ }^{86}$ entre los que se conserva un montaje que muestra el proceso de dibujo y de trabajo previo. ${ }^{87}$

El MPN contaba también en sus colecciones con una sección de objetos similares a los de algunas secciones del MNAI, como las labores de aguja. Y entre el material de enseñanza había bordados anteriores al siglo XVII (Inv. 551-558), del siglo XVII (Inv. 559-584), del siglo XVIII (Inv. 565-582), y del siglo XIX (Inv. 583-587). ${ }^{88}$ También dibujos, tiras bordadas, estarcidos para bordado (s. XVI), una bolsa y caja de evangelios bordados (s. XVIII), bordados de distintas instituciones inglesas; encajes

\footnotetext{
${ }^{84}$ Museo Pedagógico de Instrucción Primaria, Catálogo Provisional, XX-XXI.

85 Villalba, «Rafael Doménech y el Museo Nacional de Artes Decorativas», 52-53.

${ }^{86}$ Información facilitada por Ana Cabrera Lafuente, actualmente conservadora del Museo del Traje y con anterioridad Jefe del Departamento de Documentación del MNAD, suposición que se basa en determinados periódicos encontrados entre la documentación del museo.

${ }^{87}$ Cabrera, «El Museo Nacional de Artes Decorativas en sus primeros años 1912-1930», 93-95.

${ }^{88}$ Museo Pedagógico de Instrucción Primaria, Catálogo Provisional, 39-43.
} 
destinados a la enseñanza de maestras que redundaría en capacitar a sus alumnas, y que formaban "series empezadas a bordar» $\mathrm{y}$ "preparadas para continuarse», entre otros. ${ }^{89}$ Hoy, gran parte de estos fondos pertenecen a la Colección Pedagógico Textil Complutense ${ }^{90}$ y forman un conjunto de unas seis mil piezas. Había, asimismo, útiles de trabajo relacionados con labores de aguja, como devanadera, rueca y huso y aspa para hacer madejas (Inv. 614-618). ${ }^{91}$

A la sección de Industria pertenecía una «colección en serie graduada de ejemplares para la enseñanza intuitiva de la confección de calzado» (Inv. 999) ${ }^{92}$ o, en el $\mathrm{n}^{\mathrm{o}}$ de inventario 1002, un «Sombrero: manufactura. Serie de ejemplares de los diferentes estados por los que va pasando su fabricación».$^{93}$ Otra pieza era la correspondiente al n ${ }^{\circ}$ Inv. 1001: «Silk of India: Muestras de Sedas crudas de la India y de su manufactura expuestas en el Museo South Kensington for Mr. Thomas Wardle, de Leek Staffordshire», ${ }^{94}$ o las Sedas de la Fábrica de Talavera, con las notas auténticas de haber servido de muestras en un encargo de vestidos para el rey Carlos III y la princesa María Luisa, mujer de Carlos IV. ${ }^{95}$ Ambos conjuntos habían sido donados por D ${ }^{\mathrm{a}}$ Emilia Gayangos de Riaño.

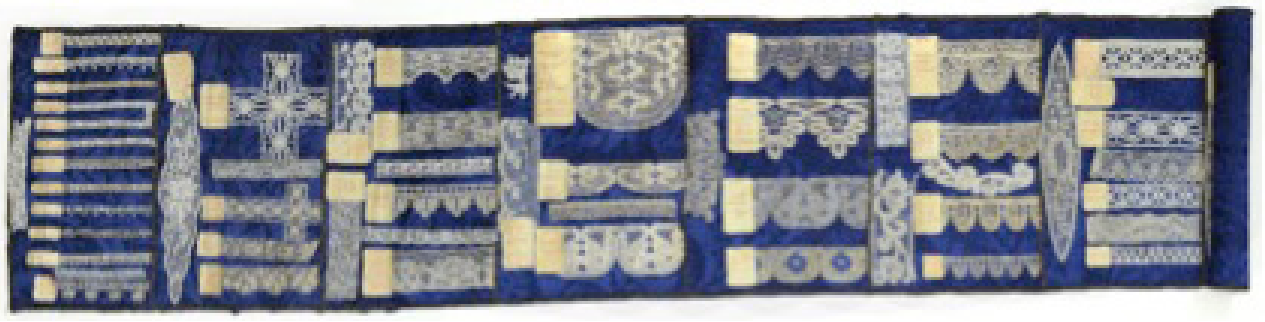

Figura 4. Serie encajes MNAD CE02742. Muestrario que fue donación de la Duquesa de Parcent. c. 1700-1800. Museo Nacional de Artes Decorativas de Madrid.

\footnotetext{
${ }^{89}$ El Catálogo recoge como material de enseñanza: Encajes de Almagro (Inv. 588), Encajes antiguos (donados por E. Gayangos, Inv. 589); Fotografías de encajes (Inv. 590-591), y Dibujos para encajes (Inv. 592). Véase Museo Pedagógico de Instrucción Primaria, Catálogo Provisional, 43-44.

${ }_{90}$ https://www.ucm.es/cultura/c.textil/ (última consulta 10-09-2018.

${ }_{91}$ Museo Pedagógico de Instrucción Primaria, Catálogo Provisional, 44.

92 Museo Pedagógico de Instrucción Primaria, Catálogo Provisional, 82-83.

${ }_{93}$ Museo Pedagógico de Instrucción Primaria, Catálogo Provisional, 85.

${ }^{94}$ Museo Pedagógico de Instrucción Primaria, Catálogo Provisional, 84-85.

${ }_{95}$ Museo Pedagógico de Instrucción Primaria, Catálogo Provisional, 84.
} 
En el MNAI merece resaltar la pieza con número de inventario CE 02742, que reúne, montado sobre damasco azul, un muestrario de encajes de motivos vegetales y geométricos, organizados por tipos y clasificados mediante etiqueta manuscrita con la procedencia, tipología y cronología. ${ }^{96}$ (figura 4). Es un muestrario donado por la Duquesa de Parcent, con varias etiquetas identificativas que nos hacen vincular con el gusto por este tipo de tejidos a personas cercanas a la ILE. Así, «Encaje español Siglos XVII al XVIII. Cedido por la Señorita de Cossío», "No 11 Encaje Arenys de Mar. Dibujo flamenco, Copia de la Colección de la Excma. Sra. Vda. de Berruete» (sic); « $\mathrm{N}^{\circ} 56$ Entredós Español Siglo XVII. Copia de uno propiedad del Sr. Weisberger»; «N $\mathrm{N}^{\circ} 34$ Encaje Duquesa. Siglo XIX cedida para la copia por la Excma. Sra. Duquesa de Parcent»; «N 88 Entredós Español. Siglo XVII. De la Colección de la Duquesa de Parcent»; «Nº 88 Encaje Español. Siglo XVI. De la Colección Pascó»; «N 69 Encaje Antiguo. De la Colección Pascó». En este listado aparecen personas vinculadas a la ILE, como la «señorita de Cossío», posiblemente Natalia Cossío, y figuras contemporáneas de pensamiento muy cercano a la ILE. Así, la Duquesa de Parcent, promotora de la Sociedad de Amigos del Arte, entidad que defendió, divulgó y propició el coleccionismo de las artes decorativas y las artes populares. ${ }^{97}$ También aparece la viuda de Aureliano de Beruete, muy cercana a Manuel Bartolomé Cossío, como demuestra su epistolario. ${ }^{98}$ Un rótulo asigna un tejido copiado de la colección de José Weissberger, figura frecuente en las exposiciones de la Sociedad de Amigos del Arte, en la sección de coleccionistas y marchantes, ${ }^{99}$ y que participó como prestador en la Exposición Lencería y encajes españoles del siglo XVI al XIX, de 1915. Se puede afirmar, en el mismo sentido, la relación estrecha entre el MNAI y la Sociedad de Amigos del Arte dada la participación del primero en las exposiciones Tejidos Españoles anteriores a la introducción del Jacquard (1917), El Abanico en España (1920) y Alfombras antiguas españolas (1933).

\footnotetext{
96 MNAD CE02742, http://ceres.mcu.es/ (última consulta (25-08-2018).

97 Prudencio Mateos Pérez, «La Sociedad Española de Amigos del Arte», Villa de Madrid IV, no. 94 (1987):70-86.

98 Javier Portus, «Manuel B. Cossío y El Greco», en El arte de saber ver. Manuel B. Cossío. La Institución Libre de Enseñanza y El Greco, 88.

99 Javier Pérez Flecha, «El marchante y coleccionista José Weissberger y la incautación y depósito de su colección en el Museo Nacional de Artes Decorativas», además de. Revista on line de artes decorativas y diseño 2 (2016): 139-152. http://www.ademasderevista.com/pdfs/ademasde_2.pdf (última consulta 14-09-2018).
} 
Las investigaciones más recientes vienen a corroborar la conexión entre el pensamiento y planteamientos de la ILE y la misión del MNAI, puesta en práctica por los miembros de su equipo técnico, liderado primero por Doménech y desde 1929 por Pérez Bueno. ${ }^{100}$

\section{PROPUESTA DE TRABAJO PARA LA PUESTA EN VALOR DE LOS OBJETOS DE COLECCIÓN DEL MNAD Y LOS MUSEOS PEDAGÓGICOS}

Pues bien, conocer los planteamientos y el catálogo provisional del MPN y ponerlo en relación con las colecciones y principios del MNAD, sugiere la elaboración de una propuesta de trabajo que diseñe una tipología de piezas con las que se puedan establecer relaciones de parentesco entre los intereses del Museo Nacional de Artes Decorativas, los intereses vinculados al mundo de la infancia y la educación y los objetos que hoy forman parte del aula y de los museos pedagógicos.

Los dos casos de los que nos hemos venido ocupando responden al tipo de museo en el que el objeto es esencial: en el caso de los museos industriales o de artes decorativas y diseño, se da una enorme importancia incluso a las piezas seriadas (aunque se dé gran valor al elemento inicial: el prototipo). Ciertamente, el interés estético es mayor en los museos de artes aplicadas. Pero el beneficio educativo y la información que nos proporciona el estudio de los objetos en relación con la historia de la educación y con el relato de nuestro patrimonio, invita a pensar en algunas tipologías de piezas, en este caso pertenecientes al MNAD, que también se exhiben en los museos pedagógicos, aunque sean susceptibles de utilización otras muchas. Me refiero, por ejemplo, a juguetes, como la casa de muñecas; a cosas de la casa: el costurero y los bordados, $\mathrm{u}$ otros objetos relacionados con las labores. O el tintero, si pensamos más en los relativos al patrimonio educativo.

\footnotetext{
100 Isabel Rodríguez Marco y Ana Cabrera Lafuente, «The Involvement of Women in the National Museum of Decorative Arts of Madrid (Spain): 1912-1942», Museum History Journal II, no. 2 (2018): 159173. DOI: 10.1080/19369816.2018.1529221; Isabel Rodríguez Marco y Ana Cabrera Lafuente, «Women involvement in the National Museum of Decorative Arts of Madrid (Spain) from 1912 to 1942 », Poster presentado en Gendering Museum Histories (Oxford, 7-8 de septiembre de 2018). Museum \& Galleries History Group Biennal Conference, 2018; Bernis y Muñoz Campos, «El museo constructor de otros contextos», 461-70; Idoia Murga, «Muros para pintar. Las artistas y la Residencia de Señoritas», en Mujeres en vanguardia. La Residencia de Señoritas en su centenario (1915-1936), eds. Almudena de la Cueva y Margarita Márquez Padorno (Madrid: Residencia de Estudiantes, 2015): 86-127.
} 
Incluso podemos vincular los fondos de colección de los museos escolares con piezas pertenecientes al MNAD: nacimientos, láminas, yesos o maquetas que, por otra parte, nos conectan con instituciones como el Museo Nacional de Reproducciones Artísticas o la Real Academia de Bellas Artes de San Fernando. Esto, sin duda, aboca a una reivindicación de las colecciones de este tipo de museos y de su utilización como herramientas educativas.

El ejemplo de las casas de muñecas, ${ }^{101}$ originarias de Núremberg en el siglo XVI, desde donde se extendieron a Holanda y al resto de Europa, surgía como un elemento útil para la educación de jóvenes y adolescentes, algo muy visual y didáctico sobre la vida cotidiana y los roles familiares. Hasta finales del siglo XVIII se entendían como «manuales de sociabilidad», pero a partir del siglo XIX se extiende su uso lúdico, fundamentalmente entre las niñas. ${ }^{102}$ Son juguetes que demuestran el avance tecnológico de la sociedad que los construye y son útiles pues rezuman valores, costumbres y modos de vida. Recientemente el Estado ha adquirido para el MNAD una casa de muñecas de mediados del siglo XIX que es, en sí misma, una evocación de la vida cotidiana de su tiempo. ${ }^{103}$ Responde a un tipo de piezas que se popularizaron a consecuencia de los ecos de la Revolución Industrial, y pasaron de ser objetos exquisitos de exposición para convertirse en objetos lúdicos, sin perder la importancia de lo estético. La «Casa de muñecas» (Inv. CE28758) (figura 5) fue realizada en Cádiz, donde vivían sus propietarios y, posiblemente, fue encargada una parte a distintos artesanos, y otra se trataría de importaciones inglesas, de gran tradición en este tipo de creaciones. Se ha transmitido de generación en generación hasta llegar al MNAD. Responde al tipo de casa burguesa decimonónica, en torno a 1825-1850, de tres plantas distribuidas en cocina comedor y cuarto de servicio en la planta baja; salón y gabinete en la planta principal, y en la planta superior, cuarto de matrimonio y tocador (figura 6).

\footnotetext{
${ }_{101}$ Heidi. A. Müller, The Nuremberg Dollhouses of the 17th Century in the Germanisches Nationalmuseum. Good Housekeeping. A domestic ideal in miniature (Nuremberg: Verl. des Germanischen Nationalmuseums, 2007).

102 Amaya Morera Villuendas, «Jugando a la Moderna», Condiciones materiales y vida cotidiana en el Antiguo Régimen», ed. Gloria Franco Rubio (Cuadernos de Historia Moderna, Anejo XIV, 2015): 135-149.

${ }^{103}$ Casa de muñecas, Inv. CE27858, Museo Nacional de Artes Decorativas (MNAD).
} 


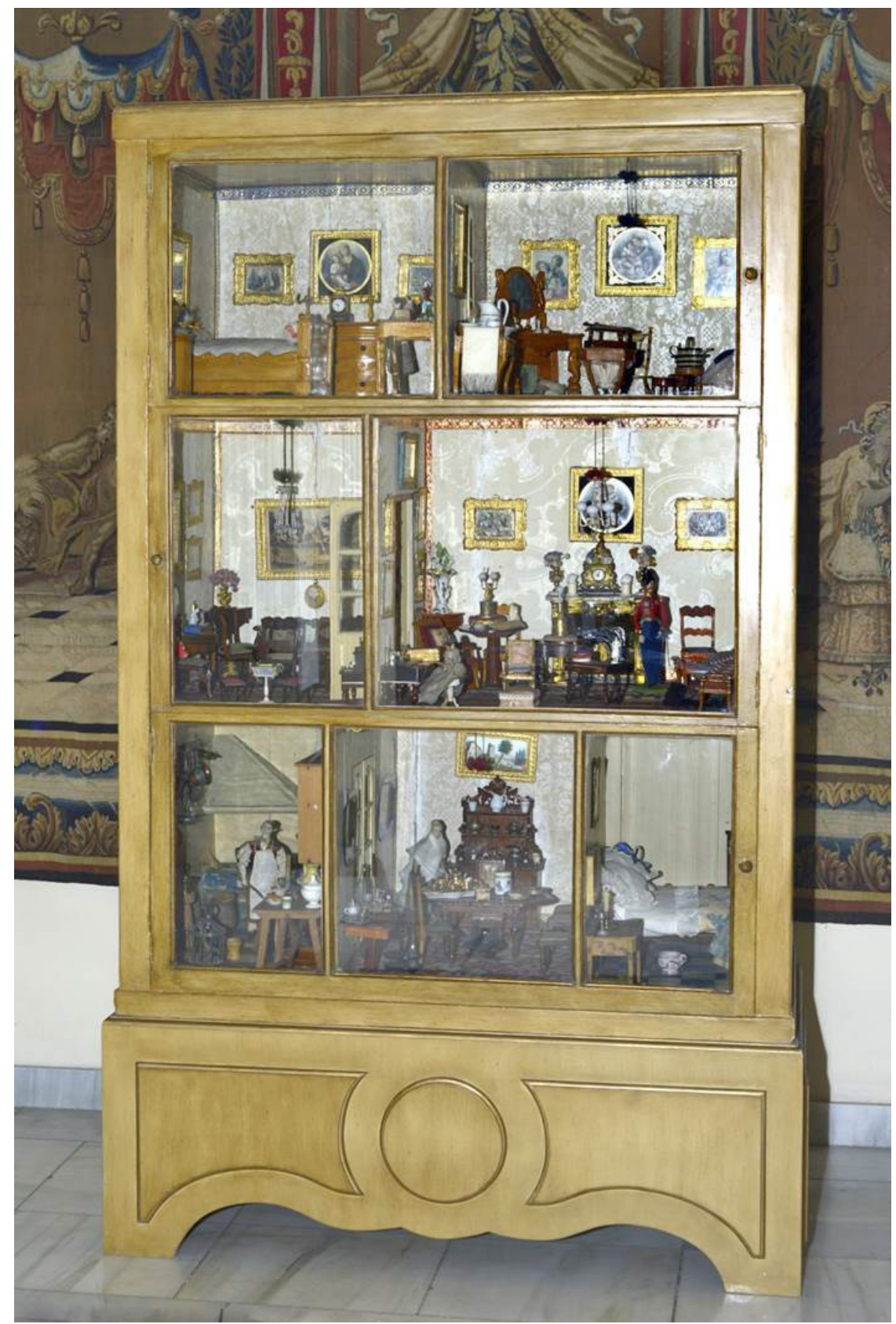

Figura 5. Casa de muñecas MNAD CE28758, c. 1840-1850. Museo Nacional de Artes Decorativas de Madrid. 


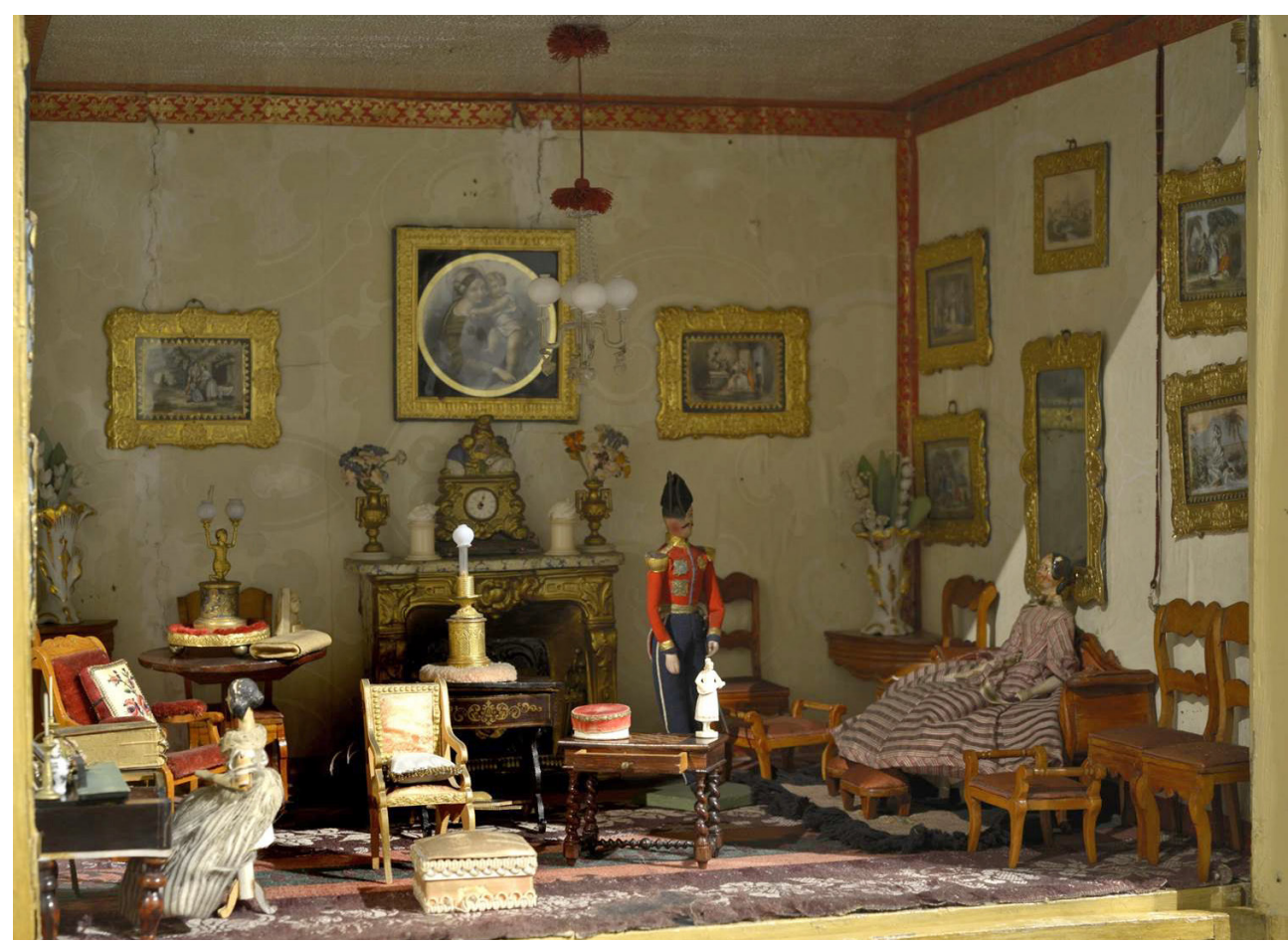

Figura 6. Casa de muñecas MNAD CE28758, c. 1840-1850. Detalle del salón. Museo Nacional de Artes Decorativas de Madrid.

Dicha casa todavía refleja costumbres del siglo XVIII, como la presencia del comedor en la planta baja y no en la principal (algo propio ya del siglo XIX). El menaje, los adornos, las estampas religiosas que reproducen obras maestras de la historia del arte, reflejan el gusto de la época y las costumbres. El gabinete femenino o sala de compañía sobresale con el escritorio, los costureros y la silla lacada. ${ }^{104}$ Todo, también la presencia de juguetes en el tocador, que indica que era una estancia de reunión para las mujeres, es signo de la función de estas casas de muñecas, que formaban parte del juego para las niñas, pero también tenían un sentido pedagógico y de conexión con el mundo de los mayores y los

104 Información facilitada por el MNAD, procedente del Informe realizado por Sofía Rodríguez Bernis, directora del Museo, con motivo del ingreso de esta pieza en la colección en el año 2015. 
valores de la época. ${ }^{105}$ Es importante decir que el propio museo posee piezas en su colección que podrían ponerse en relación con la decoración de la casa, que corroboran cómo esta es un espejo de su tiempo.

Otros museos estatales cuentan con ejemplos significativos de casas de muñecas: el Museo del Traje (CE082925, CE091180, CE109471), el Museo del Romanticismo (CE1917) y el Museo Nacional de Cerámica González Martí (CE1917). Igualmente las colecciones del Museo Pedagógico y Museo del Niño de Castilla La Mancha (Albacete), que cuenta con una sala dedicada al juego y al juguete desde comienzos del XIX al XX, ${ }^{106}$ y el Museo Laboratorio de Historia de la Educación «Manuel Bartolomé Cossío» (Madrid), muestran como se trata de un juguete vigente en nuestros días.

Podemos añadir otros objetos coincidentes que hemos detectado en las colecciones del MPN y el MNAD: la rueca (CE02362) y el huso (CE02836), que formaban parte del inventario del primero catalogados en el apartado de Labores de aguja/Útiles de trabajo: "Inv. 617: Rueca y huso dispuestas para hilar», relacionados con las devanaderas (Inv. 614 y 615), del siglo XVIII (estilo barroco) y del siglo XIX (estilo Imperio), respectivamente. Son piezas que sugieren la posibilidad de trabajar en aquellos temas relacionados con las profesiones y los oficios, pero también con las grandes obras de la Historia del Arte, como es el caso de Las Hilanderas (1655-1660) de Velázquez, o La reina doña Juana la Loca, recluida en Tordesillas con su hija, la infanta doña Catalina (1906) de Pradilla. Otro objeto que puede ser de interés y que es común a las dos tipologías de museos es el tintero, del que también encontramos en el inventario de bienes del MPN dos piezas de loza de Talavera del siglo XVIII (Invs. 526 y 5279), ${ }^{107}$ uno de ellos donado por Juan Francisco Riaño. En el MNAD, del siglo XVIII, el CE09335, de Talavera de la Reina, y el CE09328, de Emilio Niveiro, entre otros, de la fábrica de El Carmen, ambos idóneos para ser estudiados en relación con la Historia del Arte,

\footnotetext{
${ }^{105}$ De hecho, los dueños de la casa relataron a la dirección del MNAD como en la familia se había mantenido la tradición, a través de generaciones, de que las niñas fueran a ver la casa de muñecas a última hora del día y allí hacer comentarios sobre las estancias, objetos, personajes, etc. (información facilitada por Sofía Rodríguez Bernis al inicio este trabajo).

106 http://www.museodelnino.es/sala-viii/ (última consulta 25-08-2018).

107 Museo Pedagógico de Instrucción Primaria, Catálogo Provisional, 37.
} 
al ser en ocasiones objetos que encontramos en obras pictóricas y que nos hablan del gusto predominante en los inicios del siglo XX en España. Entonces se recuperaban objetos y decoraciones de los siglos XVII y XVIII español, como consecuencia de la corriente regeneracionista y de identidad nacional que provocó el resurgir de las artes decorativas españolas. ${ }^{108}$

Podríamos decir que los responsables del MPN y del MNAI actuaban con el mismo objetivo y con principios muy similares y comunes, en los que se percibía el espíritu de la ILE, el amor al trabajo bien hecho y a la esencialidad de lo popular. La atención a los textiles iniciada por Cossío dio carta de naturaleza, junto con los escritos de los hermanos Giner de los Ríos, a la presencia de las artes populares en la historiografía artística.

\section{A MODO DE CONCLUSIÓN}

La revisión de la puesta en marcha del Museo de Instrucción Primaria, después Museo Pedagógico Nacional, y del Museo Nacional de Artes Industriales, delata un paralelismo real en el tiempo y una relación estrecha en sus planteamientos desde los orígenes: en la utilización didáctica de sus colecciones, en los elementos coincidentes en los fondos custodiados por ambas entidades, en la relevante misión de una biblioteca activa en la institución, y en el apoyo y espíritu que se respiraba y que partía de la Institución Libre de Enseñanza (si bien no en la misma medida).

Son museos que indudablemente, y de manera importante, han contribuido a la creación de la modernidad en nuestro país y a la historia de la educación, por lo que es planteable la posibilidad de traer a nuestros días esta relación estableciendo conexiones entre los fondos conservados actualmente en ellos y los museos pedagógicos españoles, que son espacios vivos encargados de la conservación y divulgación del patrimonio educativo, material e inmaterial. Son espacios abiertos y dinámicos que

\footnotetext{
108 Como señala Abraham Rubio, el MNAD conserva un grupo de diecinueve piezas adquiridas en la Exposición Nacional de Artes Decorativas de 1913. Eran piezas de Emilio Niveiro (1858-1939), director de la fábrica de El Carmen de Talavera de la Reina, bajo cuya dirección la fábrica inició una etapa en la que se recuperaban modelos y decoraciones procedentes del Barroco español. Véase Abraham Rubio, Viaje a través de la cerámica artística española en el Museo Nacional de Artes Decorativas (Madrid: Ministerio de Educación, Cultura y Deporte, Secretaría General Técnica, Subdirección General de Documentación y Publicaciones, y Asociación de Amigos del Museo Nacional de Artes Decorativas, 2016), 35.
} 
favorecen las funciones propias de las instituciones museísticas: la investigación, la catalogación, la difusión, la exposición y la adquisición, pues, como señala Pablo Álvarez Domínguez, están llamados a la dinamización. ${ }^{109}$ Así, un modo de activarlos es buscar los nexos con las colecciones de artes decorativas, de museos como el MNAD, junto al cual otros nos pueden ayudar a establecer metodologías en préstamo a la hora de abordar el estudio de los objetos del patrimonio educativo: el Museo del Romanticismo, el Museo del Traje, el Museo Nacional de Antropología, el Museo Nacional de Cerámica González Martí, o las casas museo: Museo Cerralbo, Museo Sorolla y Museo Cervantes, entre otros.

Asimismo, podemos hablar de ellos como museos inclusivos, algo tan importante hoy en día en que este tema se considera un reto en el mundo de la museología. Ambos ejercían la importante labor de la investigación (la lectura del catálogo provisional del MPN es una muestra fehaciente de esto), pero también de la difusión a través de las actividades relacionadas con la enseñanza-aprendizaje de sus colecciones. Ambos se concibieron como museos abiertos que pretendían dar acceso a colectivos como mujeres, artífices, artesanos, obreros, campesinos, sin olvidar otros colectivos de mayor formación intelectual que se nutrieron de los fondos, bien para la formación docente, bien para la creación artística, o la documentación y estudio de la Historia del Arte.

Todos ellos reúnen objetos que son ventanas abiertas al conocimiento, que nos hablan de economía, mercado, cultura, historia del arte, arte popular, arquitectura, vida cotidiana, tradiciones y tantas cosas como para tenerlos en cuenta en el ámbito de la educación de nuestros alumnos. Desde esta aportación, y a partir de la revisión de las fuentes señaladas anteriormente y de la bibliografía especializada, se pretende mostrar un conjunto de realidades que dibujan una etapa de la historia del arte y de la educación en museos de la que se pueden extraer consecuencias y resultados de interés para la formación de los futuros docentes de las disciplinas de Historia del Arte, Ciencias Sociales e Historia de la Educación.

109 Pablo Álvarez Domínguez, «Los Museos Pedagógico en España: actualidad y perspectivas de futuro", en Los Museos Pedagógicos en España. Entre la memoria y la creatividad, coord. Pablo Álvarez Domínguez (Gijon: Trea, 2016): 21-32. 


\section{Nota sobre la autora}

María Villalba Salvador es doctora en Historia del Arte (UCM). Profesora del Departamento de Didácticas Específicas de la Facultad de Profesorado y Educación de la Universidad Autónoma de Madrid desde el año 2013, pertenece al área de Ciencias Sociales del departamento y se encarga de las asignaturas relativas a la Didáctica de la Historia del Arte. Previamente ha sido profesora de Enseñanza Secundaria Obligatoria y Bachillerato (Fundación San Pablo CEU) y de la Universidad Alfonso X El Sabio. Ha formado parte del Departamento de Historia y Teoría del Arte de la UAM (2004-2013) y del Departamento de Historia del Arte III de la UCM (2013). Ha trabajado en el Museo Nacional de Artes Decorativas (2004-2013) en las Áreas de Documentación e investigación, sobre los depósitos del museo y la catalogación de la colección de diseño contemporáneo. Es autora de publicaciones sobre educación en museos, didáctica de la historia del arte y del patrimonio, historia del Museo Nacional de Artes Decorativas y crítica de arte, entre otras: ;Vamos al Museo! Guías y recursos didácticos para visitar los museos (Narcea 1998), Por amor al arte (SM, 2000), Viaje a través de las artes decorativas y el diseño. Siete siglos de historia y cultura artística (coord.), (Ministerio de Cultura y Asociación de Amigos del Museo Nacional de Artes Decorativas, 2012). Es miembro de la Asociación Española de Museólogos (AEM) y secretaria de la Asociación de Amigos del Museo Nacional de Artes Decorativas.

\section{REFERENCIAS}

Alcántara, Francisco «Política artística». El Imparcial XLII, no. 14884 (21 de agosto de 1908): 3.

Álvarez Domínguez, Pablo. «Los Museos Pedagógicos en España: actualidad y perspectivas de futuro». En Los Museos Pedagógicos en España. Entre la memoria y la creatividad, coordinado por Pablo Álvarez Martínez, 21-32. Gijón: Trea, 2016.

Alzola Minondo, Pablo. El arte industrial en España. Bilbao: Casa de la Misericordia, 1892. http://bvpb.mcu.es/es/consulta/registro. $\mathrm{cmd}$ ? id $=411560$

Bolaños, María. Historia de los museos en España. Memoria, cultura y sociedad. Gijón: Trea, 1997.

Caballero, María Rosario. Inicios de la historia del arte en España: la Institución Libre de Enseñanza (1876-1936). Madrid: CSIC, Instituto de Historia, Departamento de Historia del Arte, 2002. 
Caballero, María Rosario. «El primer Manual de Historia del Arte con destino al Bachillerato. Su autor: Hermenegildo Giner de los Ríos». Imafronte 15 (2000-2001): 17-28. http://revistas.um.es/imafronte

Caballero, María Rosario. «La Historia del Arte en la enseñanza secundaria con destino al Bachillerato. Perspectiva histórica y posibilidades de futuro». Imafronte 8 y 9 (1992-93): 51-60. https://dialnet.unirioja.es/servlet/articulo?codigo $=233380$

Cabrera Lafuente, Ana. «El Museo Nacional de Artes Decorativas en sus primeros años». además de. Revista on line de artes decorativas y diseño 1 (2015): 89-101, http://www.ademasderevista.com/pdfs/numero1/articulo_cabrera. pdf

Cabrera Lafuente, Ana y María Villalba Salvador. «El Museo Nacional de Artes Decorativas (Madrid). De Museo Industrial a Museo Nacional de Artes Industriales (1850-1912). Antecedentes para la historia del Museo». RdeM. Revista de Museología 30-31 (2004): 81-88.

Canalda, Silvia, y Albert Martí. Los objetos hablan. Dossier para profesores. Madrid: Museo Nacional del Prado, Obra Social La Caixa, 2009.

Caparros Masegosa, Lola. «La institucionalización de las artes decorativas en España: de sección en las Exposiciones Nacionales de Bellas Artes a Exposición Nacional de Artes Decorativas (1897-1910)». Res Mobilis. Revista internacional de investigación en mobiliario y objetos decorativos» 5 , no. 5 (2016): 75-100. https://dialnet.unirioja.es/descarga/articulo/5290511.pdf

Chilvers, Ian, y Harold Osborne. The Oxford Dictionary of Art. New York: Oxford University Press, 1997.

Cossío, Manuel Bartolomé. El Greco. Madrid: Espasa Calpe, 1983, [1944].

Cossío, Manuel Bartolomé. «El Museo Pedagógico de Madrid». En Museo Pedagógico de Instrucción Primaria. Documentos para su historia. Legislación. Organización. Memoria de sus trabajos, 21-28. Madrid, Fortanet: 1886.

Cossío, Manuel Bartolomé. "Más sobre la educación estética en Inglaterra». Boletín de la Institución Libre de Enseñanza XI, no. 241 (1887): 353-354.

Cossío, Manuel Bartolomé. «Sobre la educación estética». Boletín de la Institución Libre de Enseñanza XI, no. 241 (1887): 321-322.

Cossío, Manuel Bartolomé y Santos M. Robledo. Anuario de primera enseñanza correspondiente a 1886. Madrid: Imprenta del Colegio Nacional de Sordomudos, 1887.

Doménech, Rafael. «Exposición Nacional de Bellas Artes de 1906». La Lectura. Madrid VI, no. 69 (1906): 22-24.

Doménech, Rafael. «La enseñanza superior de las industrias artísticas en Valencia». Revista Contemporánea XXVI, no. CXIX (30/07/1900): 122-131.

Doménech, Rafael. «La enseñanza superior de las industrias artísticas en Valencia». Revista Contemporánea XXVI, no. CXIX (15/08/1900): 225-243. 
Escolano Benito, Agustín. «Las Escuelas Normales. Siglo y medio de perspectiva histórica». Revista de Educación 269 (1982): 55-76.

Freixa, Mireia (ed.). Las vanguardias del siglo XIX. Barcelona: Gustavo Gili, 1982.

García Blanco, Ángela. Didáctica del museo: El descubrimiento de los objetos. Madrid: Ediciones de la Torre, 1994.

García del Dujo, Ángel. «El museo pedagógico nacional y las corrientes pedagógicas contemporáneas». Historia de la Educación, Revista Interuniversitaria 4 (1985): 169-182. http://revistas.usal.es/index.php/0212-0267/article/view/6622

García del Dujo, Ángel. «Manuel B. Cossío y el Museo Pedagógico Nacional». Bordón. Revista de Pedagogía 258 (1985): 367-384.

García Rodríguez, Fernando. "Teoría del arte: las artes decorativas en la prensa española del primer tercio de siglo». En Historiografía del Arte Español en los siglos XIX y XX. VII Jornadas de Arte, 459-470. Madrid: Alpuerto,1995.

Giner, Francisco. Estudios sobre artes industriales y Cartas literarias. En Obras Completas de Francisco Giner de los Ríos, vol. XV Madrid: Imprenta de Julio Cosano, 1926.

Giner, Francisco. Estudios sobre artes industriales. Madrid: Librería de José Jorro, 1892.

Giner de los Ríos, Hermenegildo. Teoría del Arte e Historia de las Bellas Artes en la Antigüedad. Baeza: Imp. y lib. de la Comisión general de libros, 1873.

González de Durana, Javier. Puente de Hierro para la Ría de Bilbao de D. Pablo Alzola. Bilbao: Colegio Oficial de Aparejadores y Arquitectos Técnicos de Vizcaya, 1986.

González Spresati, Carlos. «Las metamorfosis estéticas de Pérez Dolz». Boletín de la Sociedad Castellonense de Cultura V (1924): 250-255.

Guereña, Jean-Louis y Alejandro Tiana. «La educación popular». En Historia de la Educación en España contemporánea. Diez años de investigación, editado por Guereña, Jean Louis, Julio Ruiz Berrio y Alejandro Tiana Ferrer, 141171. Madrid: Ministerio de Educación y Ciencia, 1993.

Guerrero, Salvador (ed.). El arte de saber ver. Manuel B. Cossío. La Institución Libre de Enseñanza y El Greco. Madrid: Fundación Francisco Giner de los Ríos, Institución Libre de Enseñanza, 2016.

Hispanic Society of America (Nueva York), Mitchell A. Codding, María Dolores Jimenez-Blanco, Patrick Lenaghan \& Museo Nacional del Prado. Tesoros de la Hispanic Society of America: Visiones del mundo hispánico. Madrid: Museo Nacional del Prado, Nueva York: Hispanic Society of America, 2017.

Junta para Ampliación de Estudios e Investigaciones Científicas. Memoria correspondiente al año 1907. Madrid: Est. Tip. de la Imprenta de Hijos de M.Tello, 1908. http://cedros.residencia.csic.es/imagenes/Portal/ArchivoJAE/memorias/001.pdf 
Junta para Ampliación de Estudios e Investigaciones Científicas. Memoria correspondiente al año 1914 y 1915. Madrid: Imp. de Fortanet, 1916. http:/cedros.residencia.csic.es/imagenes/Portal/ArchivoJAE/memorias/005.pdf

Lucena Giraldo, Manuel. 82 objetos que cuentan un país: Una historia de España. Barcelona: Taurus, 2015.

MacGregor, Neil. La historia del mundo en 100 objetos. Barcelona: Debate, 2012.

Mateo de Castro, Javier. «El museo: un espacio educativo para la igualdad social. El caso pionero del Museo Pedagógico Nacional en España (18821941)». El futuro del pasado 8 (2017): 83-120. http://dx.doi. org/10.14516/ fdp.2016.008.001.003

Montero Pedrera, Ana María. «Origen y desarrollo de las Escuelas de Artes y Oficios». Historia de la educación 17 (1998): 319-330.

Morera Villuendas, Amaya. «Jugando a la Moderna». En Condiciones Materiales y vida cotidiana en el Antiguo Régimen, editado por Gloria Franco Rubio. Cuadernos de Historia Moderna XIV (2015): 135-149.

Müller, Heidi A. The Nuremberg Dollhouses of the 17th Century in the Germanisches Nationalmuseum. Good Housekeeping. A domestic ideal in miniature. Nuremberg: Verl. des Germanischen Nationalmuseums, 2007.

Murga, Idoia. «Muros para pintar. Las artistas y la Residencia de Señoritas». En Mujeres en vanguardia. La Residencia de Señoritas en su centenario (19151936), editado por Almudena de la Cueva y Margarita Márquez Padorno, 86-127. Madrid: Residencia de Estudiantes, 2015.

Museo Pedagógico de Instrucción Primaria. Catálogo Provisional. Madrid: Imprenta de Fortanet, 1890.

Ortega Morales, Natividad. La enseñanza-aprendizaje del arte. Una innovación educativa de la institución Libre de Enseñanza. Granada: Grupo Editorial Universitario, 2002.

Otero Urtaza, Eugenio. «Los viajes de formación de Cossío entre 1879 y 1889». En El arte de saber ver. Manuel B. Cossío. La Institución Libre de Enseñanza y El Greco, editado por Salvador Guerrero, 169-179. Madrid: Fundación Francisco Giner de los Ríos, Institución Libre de Enseñanza, 2016.

Otero Urtaza, Eugenio. Manuel Bartolomé Cossío. Trayectoria de un educador. Madrid: Publicaciones de la Residencia de Estudiantes y Consejo Superior de investigaciones Científicas, 1994.

Pérez Flecha, Javier. «El marchante y coleccionista José Weissberger y la incautación y depósito de su colección en el Museo Nacional de Artes Decorativas». además de. Revista on line de artes decorativas y diseño 2 (2016): 139152. http://www.ademasderevista.com/pdfs/ademasde_2.pdf

Portus, Javier. "Manuel B. Cossío y El Greco». En El arte de saber ver. Manuel B. Cossío. La Institución Libre de Enseñanza y El Greco, editado por Salvador 
Guerrero, 81-119. Madrid: Fundación Francisco Giner de los Ríos, Institución Libre de Enseñanza, 2016.

Portus, Javier y Jesusa Vega. Cossío, Lafuente, Gaya Nuño. El descubrimiento del arte español. Tres apasionados maestros. Madrid: Nivola, 2004.

Prina, Daniela. «Belgian decorative arts in the later nineteenth century. Needs for a National Museum and debates surrounding didactic collections in Brussels». Journal of the History of Collections 24, no. 2 (2012): 257-274

«Real Decreto de 30 de diciembre de 1912». Boletín Oficial del Ministerio de Instrucción Pública y Bellas Artes 2 (7 de enero de 1913).

«Museo Nacional de Artes Industriales. Reglamento». Boletín Oficial de Ministerio de Instrucción Pública y Bellas Artes, V (7), (23 de enero de 1914): 3-6.

Rodríguez Bernis, Sofía y Paloma Muñoz Campos García. «El museo constructor de otros contextos. Cien años del Museo Nacional de las Artes Decorativas». Anales de Historia del Arte 24, no. especial (noviembre, 2014): 461-470.

Rodríguez Bernis, Sofía. «Cossío y las artes populares». En El arte de saber ver. Manuel B. Cossío. La Institución Libre de Enseñanza y El Greco, editado por Salvador Guerrero, 181-191. Madrid: Fundación Francisco Giner de los Ríos, Institución Libre de Enseñanza, 2016.

Rodríguez Marco, Isabel y Ana Cabrera Lafuente. «Women involvement in the National Museum of Decorative Arts of Madrid (Spain) from 1912 to 1942 » Poster presentado en Gendereing Museum Histories (Ashmolean Museum, Oxford 7-8 septiembre 2018). Museum \& Galleries History Group Biennal Conference, 2016.

Rodríguez Marco, Isabel y Ana Cabrera Lafuente. «The Involvement of Women in the National Museum of Decorative Arts of Madrid (Spain): 1912-1942». Museum History Journal II, no. 2 (2018): 153-173. DOI: 10.1080/19369816.2018.1529221

Rubio, Abraham. Viaje a través de la cerámica artística española en el Museo Nacional de Artes Decorativas. Madrid: Ministerio de Educación, Cultura y Deporte, Secretaría General Técnica Subdirección General de Documentación y Publicaciones, y Asociación de Amigos del Museo Nacional de Artes Decorativas, 2016.

Ruskin, John. Las piedras de Venecia y otros ensayos sobre arte. Barcelona: Biblok Book Export, 2016.

Sánchez de Andrés, Leticia. «Manuales de origen krausista para la enseñanza de la estética y la historia del arte y de la música en los manuales de bachillerato». Arbor. Ciencia, pensamiento y cultura 187, no. 749 (2011): 535-545.

Santacana i Mestre, Joan, y Naira Llonch Molina. Manual de didáctica del objeto en el museo. Gijón: Ediciones Trea, 2012.

Seseña, Natacha. "La historiografía del arte popular en el siglo XX». En Historiografía del Arte español en los siglos XIX y XX, 449-457. Madrid: Alpuerto, 1995. 
Seseña, Natacha. «Los becarios de arte de la Junta para Ampliación de Estudios». En 1907-1987. La Junta para Ampliación de Estudios e Investigaciones Científicas 80 años después, coordinado por José Manuel Sánchez Ron, vol. II, 557-585. Madrid: CSIC, 1989. http://archivojae.edaddeplata.org/jae_app/ Taylor, Joshua C. Nineteenth-Century Theories of Art. Berkeley and Los Angeles, California: University of California Press, 1987.

Trusted, Marjorie. "In all cases of difference adopt Signor Riaño's view. Collecting Spanish decorative arts at South Kensington Museum in the late nineteenth century». Journal of the History of Collections 18, no. 2 (2006): 228236. http://jhc.oxfordjournals.org/content/18/2/225.full

Turpin, John. «The South Kensington System and the Dublin Metropolitan School of Art 1877-1900». Dublin Historical Record 36, no. 2 (1983): 42-64. http://www.jstor.org/stable/30100731

Venturi, Lionello Historia de la crítica de arte. Barcelona: Gustavo Gili, 1979.

Villalba Salvador, María. «Rafael Doménech y el Museo Nacional de Artes Decorativas. Génesis de una colección». además de. Revista on line de artes decorativas y diseño 1 (2015): 43-60. http://www.ademasderevista.com numero1_articulo3.php

Villalba Salvador, María y Ana Cabrera Lafuente. «El Museo Nacional de Artes Industriales, hoy Museo Nacional de Artes Decorativas (Madrid) (19121927)». Revista de Museología. HdM 36 (2006): 117-123.

Wainwright, Clive (edited for publication by Charlotte Gere). «The making of South Kensington Museum I. The Governnment Schools of Design and the founding Collection, 1837-51». Journal of the History of Collections 14, no. 1 (2002): 3-23.

Waterfield, Giles. «The People`s Galleries. South Kensington Museum - V\&A», https://yalebooksblog.co.uk/2015/09/03/the-peoples-galleries-south-kensington-museum-the-va/ 